\title{
Electromechanical analysis of adaptive piezoelectric energy harvester controlled by two segmented electrodes with shunt circuit networks
}

\begin{abstract}
This paper presents an adaptive power harvester using shunted piezoelectric control system with segmented electrodes. This technique has spurred new capability for widening the three simultaneous resonance frequency peaks using only a single piezoelectric laminated beam where normally previous works only provide a single peak for the resonance at the first mode. The benefit of the proposed techniques is that it provides effective and robust broadband power generation for application in self-powered wireless sensor devices. The smart structure beam with proof mass offset is considered to have the simultaneous combination between vibration-based power harvesting and shunt circuit control-based electrode segments. As a result, the system spurs new development of the two mathematical methods using electromechanical closed-boundary value techniques and Ritz method-based weak form analytical approach. The two methods have been used for comparison giving accurate results. For different electrode length using certain parametric tuning and harvesting circuit systems, the technique enables the predictions of the power harvesting that can be further proved to identify the performance of the system using the effect of varying circuit parameters so as to visualize the frequency and time waveform responses.
\end{abstract}

Keywords: Adaptive response - control · energy harvesting · piezoelectric · shunt circuit · smart structures $\cdot$ vibration.

\section{Introduction}

Emerging micro-power harvesters have become important due to increasing demands of portable power electronic devices that still traditionally rely on their electrical energy from battery and powerline systems. Such micro-power harvesting devices can alleviate those essential technical issues by converting the vibration energy into the usable electrical energy so as to recharge battery and enable wireless sensor devices [1]-[3]. The most common micro-power harvester has been increasingly found in a wide range of applications using thermoelectric [4], electrostatic [5], electromagnetic [6]-[7], and piezoelectric [8]-[10] transductions. In terms of advantages over other competing transducers, the piezoelectric component has high sensitivity and power density, compact design, and scalability. Some preliminary technical aspects of the piezoelectric structures with different applications have been formulated using the mathematical studies for discussing the actuated system [11]-[12], shape control system [13]-[14], thermoelastic effect [15]-[16], feedback gain control system [17]-[19], and electrical shunt control systems [20]-[24]. Typical piezoelectric power harvesters have mainly used laminate cantilever beams (unimorph or bimorph structures) because they provide high elemental strain from the transverse bending motion

M.F. Lumentut · I.M. Howard

Laboratory for Dynamic Systems of Smart Structure and Vibration,

Department of Mechanical Engineering, Curtin University, Perth, Australia

m.lumentut@curtin.edu.au ; m.lumentut@outlook.com 
to create significant electrical energy due to electric field generated from the piezoelectric element. Nevertheless, the complete process for designing robust power harvesters is very challenging since it depends on the application, geometrical parameters, physical properties, optimization, fabrication, and electronic power management circuits with sensor systems. However, the investigation of the system models using mathematical studies has become an essential feature for power harvesting research. For that reason, broader power harvesting systems have been investigated using different technical aspects where the fundamental resonance of the system has become the main aspect of producing a single peak of power harvesting amplitude. Starting with the equivalent electromechanical lumped parameter model-based closed circuit system, the standard methods using resistive impedance [8] and AC-DC rectification [25] have been used for analyzing the power harvesting techniques. Then, the system model has been continually developed using synchronized switching harvesting on an inductor (SSHI) [26]-[28] in order to create the constant reversed peak voltage for certain time waveforms giving more stable piecewise power harvesting output.

Other theoretical strategies for analyzing the power harvesting piezoelectric beam models connected with resistive circuit can also be found with the mechanical tuning system. The attached tip mass of the smart cantilever beam structure can be used to shift the single resonance frequency and increase the power output. The solution techniques have mainly focused on various theoretical implementations such as Rayleigh-Ritz method [29]-[30], distributed parameter system [31], electromechanical weak form [32], closed form methods [10],[33]-[34], assumed-mode method [9], transfer matrix [35], electromechanical finite element analysis [10],[36], and analytical voltage- and charge-type formulation techniques [37]. Alternatively, the multifrequency tuning system has been developed using the electrically connected multiple piezoelectric bimorph beams [38]-[41]. The use of multiple piezoelectric beams can generate multiple resonance peaks. The techniques can be used for matching the particular frequency from the piezoelectric structure with the vibration environment that can change over time. There is also the distinct application of using the piezoelectric components e.g. the shunt control system for the vibration suppression of the smart structures [21],[24],[42]-[43] in many different case studies. Nevertheless, it gives direct relevancy and basis for developing new power harvesting techniques as proposed in this paper. The techniques provide the adaptive response system using multiple tuning and harvesting circuits onto separated piezoelectric layers in order to widen the frequency band and stimulate multiresonance peaks while using a single piezoelectric beam.

In this paper, the piezoelectric laminated structure with proof mass offset under input base excitation was used to model the coupled system of multiple electrical shunted control and electromechanical power harvester. Novel analytical techniques of the system have been developed using the extended Hamiltonian principle for deriving the dynamical closed-form boundary value equations and Ritz method-based weak form analytical approach. The two methods have been used for comparison giving accurate results. Recently, new adaptive power harvesting response [44] has been presented. However, this paper further extends and reveals key technical equations for widening the frequency band of the three simultaneous resonance peaks while using only a single piezoelectric bimorph beam. At this stage, there are no previous works developing the proposed analytical techniques with the combinations of the multi-tuning and harvesting circuits, mechanical system (elasticity with mechanical stress and dynamic motions), and electromechanical system (electrical displacement, electrical stress and electric-polarity field). As a result of these combined techniques, the normalized closed-form electromechanical transverse dynamic equations were reduced to formulate two complete forms of electromechanical multi-mode FRFs and time waveform responses using the Laplace transform. Moreover, the reduced electromechanical transverse dynamic equations-based Ritz method using weak form technique were developed to formulate multi-mode FRFs. Certain parametric tuning and harvesting circuit system case studies are developed for predictions of the power harvesting so as to identify the performance of the system using the effect of varying circuit parameters.

\section{Constitutive Electromechanical Equations}

In Fig. 1, the robust smart structure system-based Euler-Bernoulli piezoelectric laminate beam model with proof mass offset under base excitation consists of tuning piezoelectric, substructure and harvesting piezoelectric components. On the lower layer, two electrical shunt control systems using variable $R L C$ circuits are connected separately to the etched electrodes on the tuning piezoelectric component. For the upper layer, the harvesting circuit is connected to the harvesting piezoelectric component for generating power. The middle layer is the passive substructure made from brass. The mathematical expressions for the system can be found in the forthcoming section. 


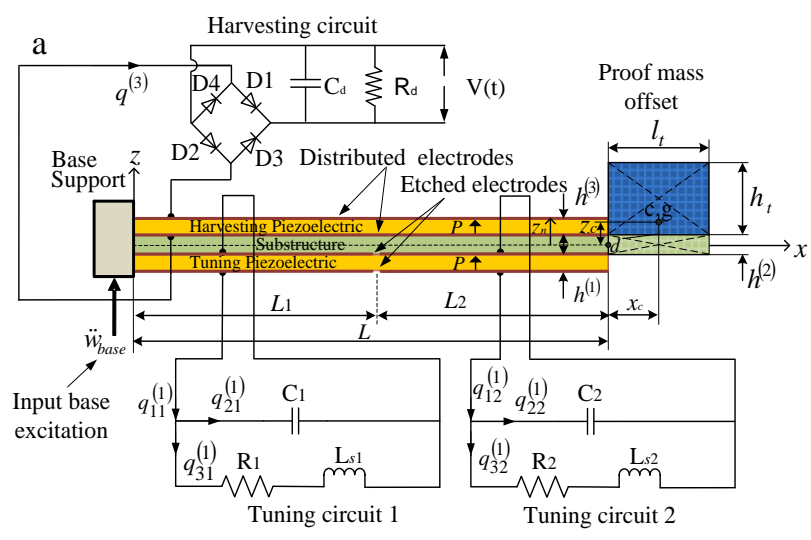

b Equivalent Tuning Piezo Simplified Equivalent Tuning Piezo

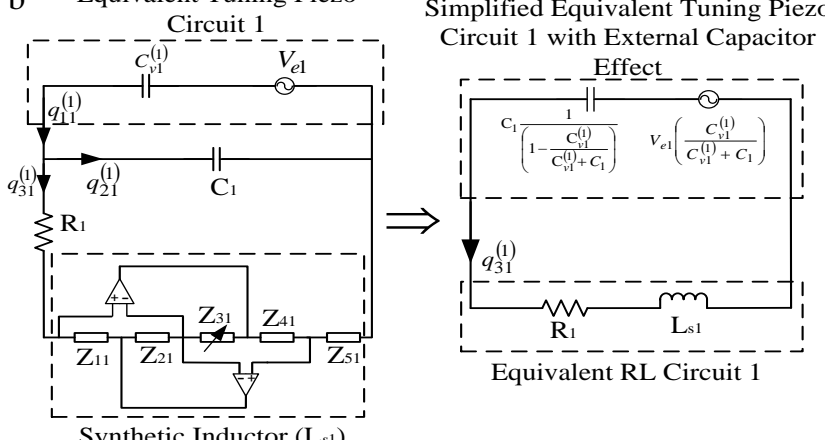

$\begin{array}{cc}\text { Equivalent Tuning Piezo } & \text { Simplified Equivalent Tuning Piezo } \\ \text { Circuit } 2 \text { with External Capacitor }\end{array}$

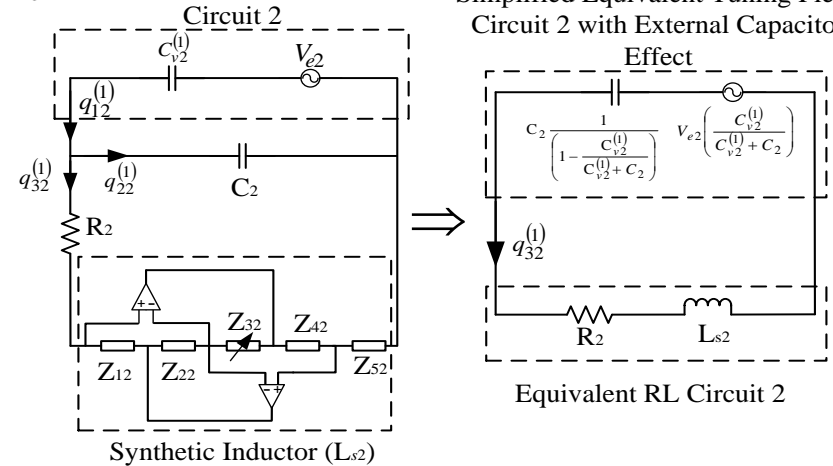

Fig.1. Piezoelectric beam power harvesting with offset proof mass operating under base input excitation: a) physical system, b) equivalent tuning circuit 1 for tuning piezoelectric layer, c) equivalent tuning circuit 2 for tuning piezoelectric layer.

It is noted here that most common piezoelectric constitutive equations that have been used for the power harvesting scheme are based on the electrical enthalpy of the continuum thermopiezoelectricity concept giving the stress-electric displacement relation. However, the electromechanical dynamic equations from the combined techniques between piezoelectric laminates and shunt and harvesting circuit systems can be formulated simultaneously according to the Helmholtz free energy [44]. The linear tuning piezoelectric constitutive equation-based Helmholtz free energy can be formulated in terms of stress-electric field relations based on the 3-1 mode of piezoelectric constant operation and 3-3 effect of piezoelectric impermittivity [45]-[46] as,

$$
\begin{gathered}
T_{1}^{(1)}=\bar{c}_{D}^{(1)} S_{1}^{(1)}-g_{31}^{(1)} D_{3}^{(1)}, \\
E_{3}^{(1)}=-g_{31}^{(1)} S_{1}^{(1)}+\varepsilon_{33}^{S^{-1} D_{3}^{(1)} .}
\end{gathered}
$$

where the parameters $T, S, E$ and $D$ represent stress, strain, electric field, and electric displacement, respectively. Coefficients $c_{D}, g$ and $\varepsilon$ indicate modified elastic constant and modified piezoelectric constant, and permittivity at constant strain respectively. Detail of each 
coefficient can be seen in Appendix A. Note that for notations, each layer from the laminated structure in Fig. 1a can be stated in the superscripts 1, 2 and 3 representing tuning piezoelectric, brass, and harvesting piezoelectric, respectively.

The modified constitutive equations in terms of stress-electric field relations for the harvesting piezoelectric material can be formulated as,

$$
\begin{aligned}
& T_{1}^{(3)}=\bar{c}_{D}^{(3)} S_{1}^{(3)}-g_{31}^{(3)} D_{3}^{(3)}, \\
& E_{3}^{(3)}=-g_{31}^{(3)} S_{1}^{(3)}+\varepsilon_{33}^{S^{-1}} D_{3}^{(3)} .
\end{aligned}
$$

The linear-elastic constitutive relation for the substructure can also be formulated as,

$$
T_{1}^{(2)}=\bar{c}_{11}^{(2)} S_{1}^{(2)}
$$

Here, the strain field for each layer of the beam can be formulated as,

$$
S_{1}(x, t)=-z \frac{\partial^{2} w(x, t)}{\partial x^{2}}
$$

where variable $z$ is the distance from the neutral axis to each layer.

\section{Electromechanical Closed-Form Boundary Value Method}

Development of analytical method is discussed in this section by combining the tuning and harvesting circuits, mechanical system (elasticity with mechanical stress and dynamic motions) and electromechanical system (electrical displacement, electrical stress and electric-polarity field). Key equations of the two analytical coupled systems for shunt tuning circuit with standard resistive circuit power harvester (non-rectifier) and AC-DC interface circuit are given in the next section.

\subsection{Coupled system of shunt tuning circuit and standard harvesting AC circuit}

The system tuning response of power harvesting devices with tip mass offset can be formulated using the extended charge type-based Hamiltonian principle to give,

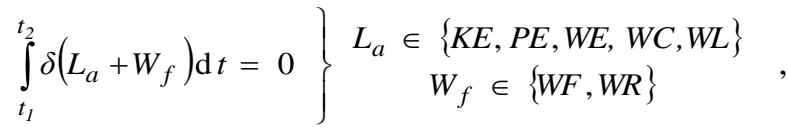

$$
\begin{aligned}
& \text { or } \int_{t_{1}}^{t_{2}}(\delta K E-\delta P E-\delta W E+\delta W F-\delta W C+\delta W L+\delta W R) \mathrm{d} t=0 .
\end{aligned}
$$

Each term of (5) can be formulated in (7)-(13). Note that detail discussion for formulating the charge type-based Hamiltonian principle can be found in [37]. The kinetic energy of the smart structure with the proof mass offset can be reformulated as,

$$
\begin{gathered}
K E=\frac{1}{2} \int_{0}^{L} \int_{A^{(1)}} \rho^{(1)} \dot{w}(x, t)^{2} \mathrm{~d} A^{(1)} \mathrm{d} x+\frac{1}{2} \int_{0}^{L} \int_{A^{(2)}} \rho^{(2)} \dot{w}(x, t)^{2} \mathrm{~d} A^{(2)} \mathrm{d} x \\
+\frac{1}{2} \int_{0}^{L} \int_{A} \rho^{(3)} \dot{w}(x, t)^{2} \mathrm{~d} A^{(3)} \mathrm{d} x+I_{0}^{t i p} x_{c} \dot{w}(L, t) \dot{\theta}(L, t)+\frac{1}{2} I_{0}^{t i p} \dot{w}(L, t)^{2}+\frac{1}{2} I_{2}^{t i p} \dot{\theta}(L, t)^{2} .
\end{gathered}
$$

Details of the mathematical equations of the dynamical piezoelectric beam and proof mass offset as shown in the kinetic energy can be found in [36]. Moreover, parameters of zeroth and second mass moment of inertias of tip mass offset $I_{0}^{t i p}$ and $I_{2}^{t i p}$ can also be found in [36]. The potential energy or strain energy of the smart structure can be formulated using the first part of (1) and (2) associated with (3) and (4) as,

$$
P E=\frac{1}{2} \int_{0}^{L} \int_{A^{(1)}} S_{1}^{(1)} T_{1}^{(1)} \mathrm{d} A^{(1)} \mathrm{d} x+\frac{1}{2} \int_{0}^{L} \int_{A^{(2)}} S_{1}^{(2)} T_{1}^{(2)} \mathrm{d} A^{(2)} \mathrm{d} x+\frac{1}{2} \int_{0}^{L} \int_{A^{(3)}} S_{1}^{(3)} T_{1}^{(3)} \mathrm{d} A^{(3)} \mathrm{d} x
$$




$$
\begin{aligned}
= & \frac{1}{2} \int_{0}^{L} \int_{A^{(1)}}\left(\bar{c}_{D}^{(1)} S_{1}^{(1)}-\sum_{h=1}^{2} g_{31}^{(1)} D_{3}^{(1)} G_{h}(x)\right) S_{1}^{(1)} \mathrm{d} A^{(1)} \mathrm{d} x \\
& +\frac{1}{2} \int_{A_{A}(2)}^{L} \int_{c_{11}^{(2)}} S_{1}^{(2)} \mathrm{d} A^{(2)} \mathrm{d} x+\frac{1}{2} \int_{A_{A}^{(3)}}^{L} \int_{C^{(3)}}\left(\bar{c}_{D}^{(3)} S_{1}^{(3)}-g_{31}^{(3)} D_{3}^{(3)}\right) S_{1}^{(3)} \mathrm{d} A^{(3)} \mathrm{d} x .
\end{aligned}
$$

Note that Heaviside functions for $G_{1}(x)=H(x)-H\left(x-L_{1}\right)$ and $G_{2}(x)=H\left(x-L_{1}\right)-H(x-L)$ are introduced due to using two segmented electrodes on the tuning piezoelectric layer. The electrical energy for the piezoelectric elements can be formulated using the second part of (1) and (2) to give,

$$
\begin{aligned}
W E= & \frac{1}{2} \int_{0}^{L} \int_{A^{(1)}} E_{3}^{(1)} D_{3}^{(1)} \mathrm{d} A^{(1)} \mathrm{d} x+\frac{1}{2} \int_{0}^{L} \int_{A^{(3)}} E_{3}^{(3)} D_{3}^{(3)} \mathrm{d} A^{(3)} \mathrm{d} x \\
= & \frac{1}{2} \int_{0}^{L} \int_{A^{(1)}} \sum_{h=1}^{2}\left(-g_{31}^{(1)} S_{1}^{(1)}+\varepsilon_{33}^{S} D_{3}^{-1} D^{(1)}\right) G_{h}(x) D_{3}^{(1)} \mathrm{d} A^{(1)} \mathrm{d} x \\
& +\frac{1}{2} \int_{0}^{L} \int_{A^{(3)}}\left(-g_{31}^{(3)} S_{1}^{(3)}+\varepsilon_{33}^{S^{-1}} D_{3}^{(3)}\right) D_{3}^{(3)} \mathrm{d} A^{(3)} \mathrm{d} x .
\end{aligned}
$$

Note that parameter $D_{3}$ in (9) can be modified for the use in the forthcoming reduced dynamic equation. For the lower layer, that can become $D_{3}^{(1)}=q_{11}^{(1)} /\left(b^{(1)} L_{1}\right) \forall G_{1}(x)$ and $D_{3}^{(1)}=q_{12}^{(1)} /\left(b^{(1)} L_{2}\right) \forall G_{2}(x)$. For the upper layer, that can show $D_{3}^{(3)}=q^{(3)} /\left(b^{(3)} L\right)$ $\forall\left(G_{1}(x)+G_{2}(x)\right)$. It is noted that unlike $D_{3}^{(1)}$, it may not be necessary to multiply $D_{3}^{(3)}$ with Heaviside functions in (8) and (9) due to the distributed electrode that meets the definite integral of the entire system itself over the interval $[0, L]$. The magnetic co-energy of the inductor in terms of tuning and harvesting circuits can be formulated as,

$$
W L=\frac{1}{2} L_{s 1} \dot{q}_{31}^{(1)}(t)^{2}+\frac{1}{2} L_{s 2} \dot{q}_{32}^{(1)}(t)^{2},
$$

where the synthetic inductance values $L_{s 1}$ and $L_{s 2}$ from Figs. 1b-c can be reduced from the equivalent impedance analysis, $Z_{i n, h}=\left(Z_{1 h} Z_{3 h} Z_{5 h}\right) /\left(Z_{2 h} Z_{4 h}\right) \forall h \in\{1,2\}$ for two segments by allowing the relations $Z_{1 h}=R_{1 h}, \quad Z_{2 h}=R_{2 h}, \quad Z_{3 h}=R_{3 h}, \quad Z_{5 h}=R_{5 h}$, and $Z_{4 h}=1 /\left(j \omega C_{s h}\right)$ to give $Z_{i n, h}=j \omega L_{s h}$ [47]-[49]. Therefore, the synthetic inductance value for tuning circuit one and two can be respectively formulated to give $L_{s 1}=\left(R_{11} R_{31} R_{51} C_{s 1}\right) / R_{21}$ and $L_{s 2}=\left(R_{12} R_{32} R_{52} C_{s 2}\right) / R_{22}$. Note that since the large inductance value for the tuning circuit is not commercially available, implementing synthetic inductance is practical which is an integrated circuit mainly consisting of Op-amp systems (e.g. 741 Op-amp circuit or OPA445 Op-amp), resistors $R_{s h}$ and capacitor $C_{s h}$ circuits [24],[48]-[49] as can be seen in Fig. 1b-c.

The electrical energy of the capacitor in terms of the tuning circuits can be formulated as,

$$
W C=\frac{1}{2 C_{1}} q_{21}^{(1)}(t)^{2}+\frac{1}{2 C_{2}} q_{22}^{(1)}(t)^{2} .
$$

The non-conservative work on the system due to the input base excitation can be stated as,

$$
\begin{aligned}
W F= & -\int_{0}^{L} \int_{A^{(1)}} \rho^{(1)} w(x, t) \mathrm{d} A^{(1)} \mathrm{d} x \ddot{w}_{\text {base }}(t)-\int_{0}^{L} \int_{A^{(2)}} \rho^{(2)} w(x, t) \mathrm{d} A^{(2)} \mathrm{d} x \ddot{w}_{\text {base }}(t) \\
& -\int_{0}^{L} \int_{A^{(3)}} \rho^{(3)} w(x, t) \mathrm{d} A^{(3)} \mathrm{d} x \ddot{w}_{\text {base }}(t)-I_{0}^{t i p} x_{c} \theta(L, t) \ddot{w}_{\text {base }}(t)-I_{0}^{t i p} w(L, t) \ddot{w}_{\text {base }}(t) .
\end{aligned}
$$

Note that details of the mathematical expression given in (12) can be found in [36].

The electrical work dissipated by resistors can be stated as,

$$
\delta W R=-R_{1} \dot{q}_{31}^{(1)}(t) \delta q_{31}^{(1)}(t)-R_{2} \dot{q}_{32}^{(1)}(t) \delta q_{32}^{(1)}(t)-R_{d} \dot{q}^{(3)}(t) \delta q^{(3)}(t) .
$$


The functional forms $L_{a}$ and $W_{f}$ from Hamiltonian's principle can be seen as the continuous differentiable functions of virtual displacement, electric displacement and charge for the whole systems that can be stated as,

$$
\begin{gathered}
L_{a}=L_{a}\left(\dot{w}(x, t), \dot{w}(L, t), \frac{\partial \dot{w}(L, t)}{\partial x}, \frac{\partial^{2} w(x, t)}{\partial x^{2}}, D_{3}^{(1)}(z, t), D_{3}^{(3)}(z, t), q_{21}^{(1)}(t), q_{22}^{(1)}(t), \dot{q}_{31}^{(1)}(t), \dot{q}_{32}^{(1)}(t)\right), \\
W_{f}=W_{f}\left(w(x, t), \frac{\partial w(L, t)}{\partial x}, w(L, t), q_{31}^{(1)}(t), q_{32}^{(1)}(t), q^{(3)}(t)\right) .
\end{gathered}
$$

Formulating (14) and (15) using total differential equations gives,

$$
\begin{gathered}
\delta L_{a}=\frac{\partial L_{a}}{\partial \dot{w}(x, t)} \delta \dot{w}(x, t)+\frac{\partial L_{a}}{\partial \dot{w}(L, t)} \delta \dot{w}(L, t)+\frac{\partial L_{a}}{\partial\left(\frac{\partial \dot{w}}{\partial x}(L, t)\right)} \delta\left(\frac{\partial \dot{w}}{\partial x}(L, t)\right)+\frac{\partial L_{a}}{\partial\left(\frac{\partial^{2} w(x, t)}{\partial x^{2}}\right)} \delta\left(\frac{\partial^{2} w(x, t)}{\partial x^{2}}\right) \\
+\frac{\partial L_{a}}{\partial D_{3}^{(1)}(z, t)} \delta D_{3}^{(1)}(z, t)+\frac{\partial L_{a}}{\partial D_{3}^{(3)}(z, t)} \delta D_{3}^{(3)}(z, t)+\frac{\partial L_{a}}{\partial q_{21}^{(1)}(t)} \delta q_{21}^{(1)}(t) \\
+\frac{\partial L_{a}}{\partial q_{22}^{(1)}(t)} \delta q_{22}^{(1)}(t)+\frac{\partial L_{a}}{\partial \dot{q}_{31}^{(1)}(t)} \delta \dot{q}_{31}^{(1)}(t)+\frac{\partial L_{a}}{\partial \dot{q}_{31}^{(1)}(t)} \delta \dot{q}_{31}^{(1)}(t), \\
\delta W_{f}=\frac{\partial W_{f}}{\partial w(x, t)} \delta w(x, t)+\frac{\partial W_{f}}{\partial\left(\frac{\partial w}{\partial x}(L, t)\right)} \delta\left(\frac{\partial w}{\partial x}(L, t)\right)+\frac{\partial W_{f}}{\partial w(L)} \delta w(L, t) \\
+\frac{\partial W_{f}}{\partial q_{31}^{(1)}(t)} \delta q_{31}^{(1)}(t)+\frac{\partial W_{f}}{\partial q_{32}^{(1)}(t)} \delta q_{32}^{(1)}(t)+\frac{\partial W_{f}}{\partial q^{(3)}(t)} \delta q^{(3)}(t) .
\end{gathered}
$$

Corresponding with (7)-(13) and (16)-(17), Eq. (6) can be further formulated using integrodifferential equations and variational principles. After simplification, the electromechanical dynamic closed-form boundary value equation for the analytical coupled system of electrical shunt control and power harvester system can be reduced to give,

$$
\begin{aligned}
& \int_{t_{1}}^{t_{2}}\left[\int _ { 0 } ^ { L } \left\{-I_{0} \ddot{w}(x, t)-I_{0} \ddot{w}_{\text {base }}(t)-C_{t} \frac{\partial^{2}}{\partial x^{2}}\left(\frac{\partial^{2} w(x, t)}{\partial x^{2}}\right)-\eta_{1}^{(1)} \frac{\partial^{2}\left(H(x)-H\left(x-L_{1}\right)\right) q_{11}^{(1)}(t)}{\partial x^{2}}\right.\right. \\
& \left.-\eta_{2}^{(1)} \frac{\partial^{2}\left(H\left(x-L_{1}\right)-H(x-L)\right) q_{12}^{(1)}(t)}{\partial x^{2}}\right\} \delta w(x, t) \mathrm{d} x-\left\{x_{c} I_{0}^{t i p} \frac{\partial \ddot{w}(L, t)}{\partial x}+I_{0}^{t i p} \ddot{w}(L, t)+I_{0}^{t i p} \ddot{w}_{\text {base }}\right\} \delta w(L, t) \\
& +\left.\left\{\eta_{1}^{(1)} \frac{\partial\left(H(x)-H\left(x-L_{1}\right)\right) q_{11}^{(1)}(t)}{\partial x}+\eta_{2}^{(1)} \frac{\partial\left(H\left(x-L_{1}\right)-H(x-L)\right) q_{12}^{(1)}(t)}{\partial x}+C_{t} \frac{\partial}{\partial x}\left(\frac{\partial^{2} w(x, t)}{\partial x^{2}}\right)\right\} \delta w(x, t)\right|_{0} ^{L} \\
& -\left\{I_{2}^{t i p} \frac{\partial \ddot{w}(L, t)}{\partial x}+x_{c} I_{0}^{(t i p)} \ddot{w}_{\text {base }}+x_{c} I_{0}^{t i p} \ddot{w}(L, t)\right\} \delta \frac{\partial w(L, t)}{\partial x}-\left\{\eta_{1}^{(1)}\left(H(x)-H\left(x-L_{1}\right)\right) q_{11}^{(1)}(t)\right. \\
& \left.+\eta_{2}^{(1)}\left(H\left(x-L_{1}\right)-H(x-L)\right) q_{12}^{(1)}(t)+\eta^{(3)} q^{(3)}(t)+C_{t} \frac{\partial^{2} w(x, t)}{\partial x^{2}}\right\}\left.\delta \frac{\partial w(x, t)}{\partial x}\right|_{0} ^{L} \\
& -\left\{\left(\int_{0}^{L} \eta_{1}^{(1)} \frac{\partial^{2} w(x, t)}{\partial x^{2}} \mathrm{~d} x+\frac{q_{11}^{(1)}(t)}{C_{v 1}^{(1)}}\right)\left(H(x)-H\left(x-L_{1}\right)\right)\right\} \delta q_{11}^{(1)}(t) \\
& -\left\{\left(\int_{0}^{L} \eta_{2}^{(1)} \frac{\partial^{2} w(x, t)}{\partial x^{2}} \mathrm{~d} x+\frac{q_{12}^{(1)}(t)}{C_{v 2}^{(1)}}\right)\left(H\left(x-L_{1}\right)-H(x-L)\right)\right\} \delta q_{12}^{(1)}(t) \\
& -\left\{\int_{0}^{L} \eta^{(3)} \frac{\partial^{2} w(x, t)}{\partial x^{2}} \mathrm{~d} x+\frac{q^{(3)}(t)}{C_{v}^{(3)}}\right\} \delta q^{(3)}(t)-\left\{L_{s 1} \ddot{q}_{31}^{(1)}(t)+R_{1} \dot{q}_{31}^{(1)}(t)\right\} \delta q_{31}^{(1)}(t)-\frac{q_{21}^{(1)}(t) \delta q_{21}^{(1)}(t)}{C_{1}} \\
& \left.-R_{d} \dot{q}^{(3)}(t) \delta q^{(3)}-\left\{L_{s 2} \ddot{q}_{32}^{(1)}(t)+R_{2} \dot{q}_{32}^{(1)}(t)\right\} \delta q_{32}^{(1)}(t)-\frac{q_{22}^{(1)}(t) \delta q_{22}^{(1)}(t)}{C_{2}}\right] \mathrm{d} t=0 \quad .
\end{aligned}
$$


Note that certain coefficients can be found in Appendices B and C. Also note that other stiffness coefficient $C_{t}$ and the zeroth mass moment of inertia of all layers $I_{0}$ can be found in [36]. Applying KCL method for the tuning circuits in Figs. 1b-c gives the electric charge equation as,

$$
q_{11}^{(1)}=q_{21}^{(1)}+q_{31}^{(1)}, \quad q_{12}^{(1)}=q_{22}^{(1)}+q_{32}^{(1)} .
$$

As shown, variables $q_{21}^{(1)}$ and $q_{22}^{(1)}$ in (18) can be eliminated in the forthcoming reduced equations for simplicity using the relation as,

$$
\begin{aligned}
& \frac{q_{21}^{(1)}(t)}{C_{1}} \delta q_{21}^{(1)}(t)=\frac{q_{11}^{(1)}(t)}{C_{1}} \delta q_{11}^{(1)}(t)-\frac{q_{31}^{(1)}(t)}{C_{1}} \delta q_{11}^{(1)}(t)-\frac{q_{11}^{(1)}(t)}{C_{1}} \delta q_{31}^{(1)}(t)+\frac{q_{31}^{(1)}(t)}{C_{1}} \delta q_{31}^{(1)}(t), \\
& \frac{q_{22}^{(1)}(t)}{C_{2}} \delta q_{22}^{(1)}(t)=\frac{q_{12}^{(1)}(t)}{C_{2}} \delta q_{12}^{(1)}(t)-\frac{q_{32}^{(1)}(t)}{C_{2}} \delta q_{12}^{(1)}(t)-\frac{q_{12}^{(1)}(t)}{C_{2}} \delta q_{32}^{(1)}(t)+\frac{q_{32}^{(1)}(t)}{C_{2}} \delta q_{32}^{(1)}(t) .
\end{aligned}
$$

After applying the mathematical lemma of duBois-Reymond's theorem for each virtual displacement field, the first constitutive electromechanical dynamic equation can be formulated as,

$$
\begin{aligned}
\delta w(x, t): I_{0} \ddot{w}(x, t)+I_{0} \ddot{w}_{\text {base }}(t)+ & C_{t} \frac{\partial^{2}}{\partial x^{2}}\left(\frac{\partial^{2} w(x, t)}{\partial x^{2}}\right)+\eta_{1}^{(1)} \frac{\partial^{2}\left(H(x)-H\left(x-L_{1}\right)\right) q_{11}^{(1)}(t)}{\partial x^{2}} \\
& +\eta_{2}^{(1)} \frac{\partial^{2}\left(H\left(x-L_{1}\right)-H(x-L)\right) q_{12}^{(1)}(t)}{\partial x^{2}}=0 .
\end{aligned}
$$

The second, third, fourth, fifth, and sixth constitutive electromechanical dynamic equations related to the tuning and harvesting circuits can be formulated respectively as,

$$
\begin{aligned}
& \delta q_{11}^{(1)}(t): \int_{0}^{L} \eta_{1}^{(1)}\left(H(x)-H\left(x-L_{1}\right)\right) \frac{\partial^{2} w(x, t)}{\partial x^{2}} \mathrm{~d} x \\
& +\left(\frac{\left(H(x)-H\left(x-L_{1}\right)\right)}{C_{v 1}^{(1)}}+\frac{1}{C_{1}}\right) q_{11}^{(1)}(t)-\frac{q_{31}^{(1)}(t)}{C_{1}}=0, \\
& \delta q_{31}^{(1)}(t): L_{s 1} \ddot{q}_{31}^{(1)}(t)+R_{1} \dot{q}_{31}^{(1)}(t)+\frac{q_{31}^{(1)}(t)}{C_{1}}-\frac{q_{11}^{(1)}(t)}{C_{1}}=0, \\
& q_{12}^{(1)}(t): \int_{0}^{L} \eta_{2}^{(1)}\left(H\left(x-L_{1}\right)-H(x-L)\right) \frac{\partial^{2} w(x, t)}{\partial x^{2}} \mathrm{~d} x \\
& +\left(\frac{\left(H\left(x-L_{1}\right)-H(x-L)\right)}{C_{v 2}^{(1)}}+\frac{1}{C_{2}}\right) q_{12}^{(1)}(t)-\frac{q_{32}^{(1)}(t)}{C_{2}}=0, \\
& \delta q_{32}^{(1)}(t): L_{s 2} \ddot{q}_{32}^{(1)}(t)+R_{2} \dot{q}_{32}^{(1)}(t)+\frac{q_{32}^{(1)}(t)}{C_{2}}-\frac{q_{12}^{(1)}(t)}{C_{2}}=0, \\
& \delta q^{(3)}(t): \int_{0}^{L} \eta^{(3)} \frac{\partial^{2} w(x, t)}{\partial x^{2}} \mathrm{~d} x+\frac{q^{(3)}(t)}{C_{v}^{(3)}}+R_{d} \dot{q}^{(3)}(t)=0 .
\end{aligned}
$$

The boundary conditions can be formulated as,

$$
\begin{gathered}
w(0, t)=0 \quad, \frac{\partial w(0, t)}{\partial x}=0, \\
\delta w(L, t): x_{c} I_{0}^{t i p} \frac{\partial \ddot{w}(L, t)}{\partial x}+I_{0}^{t i p} \ddot{w}(L, t)+I_{0}^{t i p} \ddot{w}_{\text {base }}-C_{t} \frac{\partial}{\partial x}\left(\frac{\partial^{2} w(L, t)}{\partial x^{2}}\right)=0, \\
\delta \frac{\partial w(L, t)}{\partial x}: x_{c} I_{0}^{t i p} \ddot{w}_{b a s e}+x_{c} I_{0}^{t i p} \ddot{w}(L, t)+I_{2}^{t i p} \frac{\partial \ddot{w}(L, t)}{\partial x}+C_{t} \frac{\partial^{2} w(L, t)}{\partial x^{2}}+\eta^{(3)} q^{(3)}(t)=0 .
\end{gathered}
$$

The solution form of (21)-(23) can be formulated using mode superposition depending on the normalized mode shapes and generalized time dependent coordinates to give,

$$
w(x, t)=\sum_{r=1}^{\infty} \hat{W}_{r}(x) w_{r}(t) .
$$

Note that the normalized mode shape in (24) can be found in Appendix D. 
After manipulation and simplification of using (24) into (21)-(23), the normalized closed-form electromechanical transverse dynamic equations with damping effect can be reduced as,

$$
\begin{gathered}
\ddot{w}_{r}(t)+2 \zeta_{r} \omega_{r} \dot{w}_{r}(t)+\omega_{r}{ }^{2} w_{r}(t)-\sum_{r=1}^{\infty} \hat{T}_{1 r}^{(1)} T_{1 r}^{(1)} \mu_{1} C_{1} w_{r}(t) \\
-\sum_{r=1}^{\infty} \hat{T}_{2 r}^{(1)} T_{2 r}^{(1)} \mu_{2} C_{2} w_{r}(t)+T_{1 r}^{(1)} \mu_{1} q_{31}^{(1)}(t)+T_{2 r}^{(1)} \mu_{2} q_{32}^{(1)}(t) \\
+T_{r}^{(3)} q^{(3)}(t)=-Q_{r} \ddot{w}_{\text {base }}(t) .
\end{gathered}
$$

Combining (22a) and (22b) including (22c) and (22d), the results of which can be associated with (22e) using (24) as,

$$
\begin{gathered}
L_{s 1} \ddot{q}_{31}^{(1)}(t)+R_{1} \dot{q}_{31}^{(1)}(t)+P_{C 1} q_{31}^{(1)}(t)+\sum_{r=1}^{\infty} \hat{T}_{1 r}^{(1)} \mu_{1} w_{r}(t)=0, \\
L_{s 2} \ddot{q}_{32}^{(1)}(t)+R_{2} \dot{q}_{32}^{(1)}(t)+P_{C 2} q_{32}^{(1)}(t)+\sum_{r=1}^{\infty} \hat{T}_{2 r}^{(1)} \mu_{2} w_{r}(t)=0, \\
R_{d} \dot{q}^{(3)}(t)+P_{V}^{(3)} q^{(3)}(t)+\sum_{r=1}^{\infty} \hat{T}_{r}^{(3)} w_{r}(t)=0 .
\end{gathered}
$$

It is noted that Eqs. (25) and (26) consist of four coupled tuning electromechanical power harvesting equations. At this case, since Eqs. (25) and (26) have been normalized, their parameters can be reduced as,

$$
\begin{gathered}
T_{1 r}^{(1)}=\eta_{1}^{(1)} \frac{\mathrm{d} \hat{W}_{r}}{\mathrm{~d} x}\left(L_{1}\right), T_{2 r}^{(1)}=\eta_{2}^{(1)} \frac{\mathrm{d} \hat{W}_{r}\left(L-L_{1}\right)}{\mathrm{d} x}, \quad T_{r}^{(3)}=\eta^{(3)} \frac{\mathrm{d} \hat{W}_{r}}{\mathrm{~d} x}(L), \\
\sum_{r=1}^{\infty} \hat{T}_{1 r}^{(1)}=\sum_{r=1}^{\infty} \eta_{1}^{(1)} \frac{\mathrm{d} \hat{W}_{r}}{\mathrm{~d} x}\left(L_{1}\right), \quad \sum_{r=1}^{\infty} \hat{T}_{2 r}^{(1)}=\sum_{r=1}^{\infty} \eta_{2}^{(1)} \frac{\mathrm{d} \hat{W}_{r}\left(L-L_{1}\right)}{\mathrm{d} x}, \\
\sum_{r=1}^{\infty} \hat{T}_{r}^{(3)}=\sum_{r=1}^{\infty} \int_{0}^{L} \eta^{(3)} \frac{\mathrm{d}^{2} \hat{W}_{r}(x)}{\mathrm{d} x^{2}} \mathrm{~d} x, P_{V}^{(3)}=\frac{1}{C_{v}^{(3)}}, P_{C 1}=\frac{1}{C_{1}}\left(1-\mu_{1}\right), \\
P_{C 2}=\frac{1}{C_{2}}\left(1-\mu_{2}\right), \quad \mu_{1}=\frac{C_{v 1}^{(1)}}{\left(C_{v 1}^{(1)}+C_{1}\right)}, \mu_{2}=\frac{C_{v 2}^{(1)}}{\left(C_{v 2}^{(1)}+C_{2}\right)}, \\
Q_{r}=\int_{0}^{L} I_{0} \hat{W}_{r}(x) \mathrm{d} x+I_{0}^{t i p} \hat{W}_{r}(L)+x_{c} I_{0}^{t i p} \frac{\mathrm{d} \hat{W}_{r}(L)}{\mathrm{d} x} .
\end{gathered}
$$

Note that Eq. (26a) can be expressed into the series equivalent circuit as shown in Fig. 1b where parameter $V_{e 1}=\sum_{r=1}^{\infty} \hat{T}_{1 r}^{(1)} w_{r}(t)$ represents the equivalent voltage source one generated due to electromechanical piezoelectric coupling one and mechanical motion, where parameter equivalent capacitor one $C_{1} /\left(1-\mu_{1}\right)$ on the circuit represents $P_{C 1}=\left(1-\mu_{1}\right) / C_{1}$ on the equation and $\mu_{1}$ is a constant term. The similar system using (26b) can also be applied for the tuning circuit two in Fig. 1c. After simplification, Eqs. (25) and (26) can be further formulated using Laplace transformation giving the transfer functions which can be reduced into the electric charge frequency response functions (FRFs) at harvesting circuit as,

$$
\frac{q^{(3)}(j \omega)}{-\omega^{2} w_{b a s e} e^{j \omega t}}=-\frac{\sum_{r=1}^{\infty} \frac{Q_{r} \hat{T}_{r}^{(3)}}{F_{r} G}}{1-\sum_{r=1}^{\infty} \frac{\hat{T}_{1 r}^{(1)} T_{1 r}^{(1)} \mu_{1}^{2}}{F_{r} E_{1}}-\sum_{r=1}^{\infty} \frac{\hat{T}_{2 r}^{(1)} T_{2 r}^{(1)} \mu_{2}^{2}}{F_{r} E_{2}}-\sum_{r=1}^{\infty} \frac{\hat{T}_{r}^{(3)} T_{r}^{(3)}}{F_{r} G}} .
$$

where,

$$
\begin{gathered}
F_{r}=\omega_{r}{ }^{2}-\hat{T}_{1 r}^{(1)} T_{1 r}^{(1)} \mu_{1} C_{1}-\hat{T}_{2 r}^{(1)} T_{2 r}^{(1)} \mu_{2} C_{2}-\omega^{2}+j 2 \zeta_{r} \omega_{r} \omega, \quad E_{1}=P_{C 1}-L_{s 1} \omega^{2}+j \omega R_{1}, \\
E_{2}=P_{C 2}-L_{s 2} \omega^{2}+j \omega R_{2}, \quad G=P_{V}^{(3)}+j \omega R_{d} .
\end{gathered}
$$

Other multimode FRFs relation can also be further formulated by using (28). Here, voltage and power FRFs across the resistor of the harvesting circuit can be formulated respectively as, 


$$
\begin{gathered}
\frac{v_{R}^{(3)}(j \omega)}{-\omega^{2} w_{\text {base }} e^{j \omega t}}=j \omega R_{d} \frac{q^{(3)}(j \omega)}{-\omega^{2} w_{\text {base }} e^{j \omega t}}, \\
\frac{P_{R}^{(3)}(j \omega)}{\left(-\omega^{2} w_{\text {base }} e^{j \omega t}\right)^{2}}=-\omega^{2} R_{d} \frac{q^{(3)}(j \omega)^{2}}{\left(-\omega^{2} w_{\text {base }} e^{j \omega t}\right)^{2}} .
\end{gathered}
$$

\subsection{Coupled system of shunt tuning circuit and standard harvesting DC interface circuit}

During each half-cycle period, the production of output current of the harvesting piezoelectric element through the AC-DC interface circuit can be illustrated as two intervals as shown in Fig. 2.

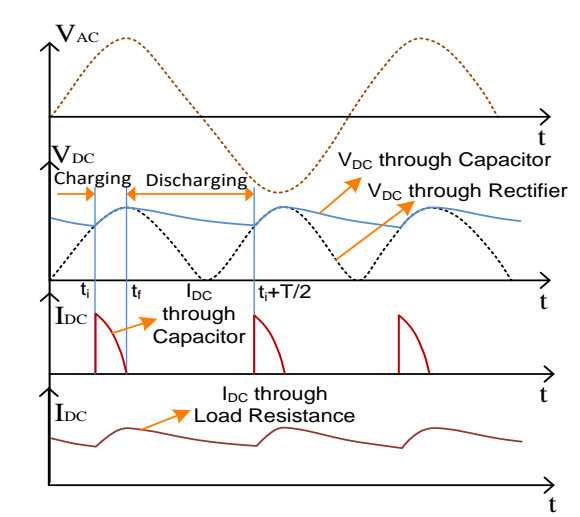

Fig.2. Time waveforms of the standard harvesting circuit

a. Current flowing with interval $t_{i}<t<t_{f}$ indicating the charging time every half-cycle of the waveform.

With the corresponding previous theoretical derivations, the following equations of coupled system response during the period of charging can be formulated using the previous equations in (25)-(26a,b). Only Eq. (26c) with slight modification of the first term gives,

$$
v_{d}+P_{V}^{(3)} q^{(3)}(t)+\sum_{r=1}^{\infty} \hat{T}_{r}^{(3)} w_{r}(t)=0
$$

Note that variable $v_{d}$ in (31) was introduced by replacing the first part from (26c). This can be obtained by removing the third term in (13) and introducing $\delta W F r=v_{d}(t) \delta q^{(3)}(t)$ in (6). Differentiating (31) with respect to time gives,

$$
\dot{v}_{d}+P_{V}^{(3)} \dot{q}^{(3)}(t)+\sum_{r=1}^{\infty} \hat{T}_{r}^{(3)} \dot{w}_{r}(t)=0 .
$$

The equation for the harvesting circuit can be formulated as,

$$
\dot{q}^{(3)}(t)-C_{d} \dot{v}_{d}-\frac{v_{d}}{R_{d}}=0 .
$$

Substituting parameter $q^{(3)}(t)$ from (31) into (25) and parameter $\dot{q}^{(3)}(t)$ from (32) into (33) and joining the results with (26a) and (26b) gives the state-space representation of the multi-mode response system, 


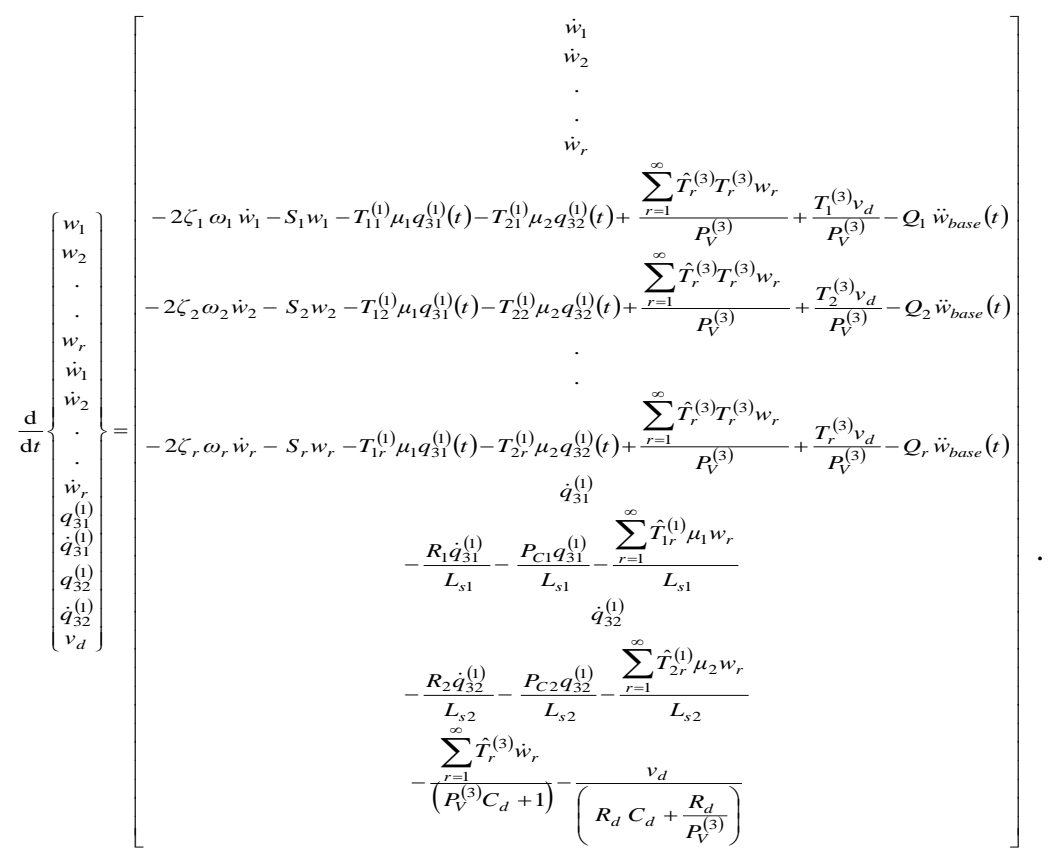

where,

$$
\begin{gathered}
S_{1}=\omega_{1}{ }^{2}-\hat{T}_{11}^{(1)} T_{11}^{(1)} \mu_{1} C_{1}-\hat{T}_{21}^{(1)} T_{21}^{(1)} \mu_{2} C_{2}, \quad S_{2}=\omega_{2}{ }^{2}-\hat{T}_{12}^{(1)} T_{12}^{(1)} \mu_{1} C_{1}-\hat{T}_{22}^{(1)} T_{22}^{(1)} \mu_{2} C_{2}, \\
S_{r}=\omega_{r}{ }^{2}-\hat{T}_{1 r}^{(1)} T_{1 r}^{(1)} \mu_{1} C_{1}-\hat{T}_{2 r}^{(1)} T_{2 r}^{(1)} \mu_{2} C_{2} .
\end{gathered}
$$

b. Current flowing with interval $t_{f}<t<t_{i}+T / 2$ indicating the discharging times every halfcycle of the waveform.

The harvesting circuit can be formulated as,

$$
C_{d} \dot{v}_{d}+\frac{v_{d}}{R_{d}}=0
$$

The solution form of (36) can be stated as,

$$
v_{d}(t)=v_{d}\left(t_{f}\right) \exp \left(\frac{-\left(t-t_{f}\right)}{C_{d} R_{d}}\right) .
$$

Note that the expressions of (34) and (37) can be utilized to estimate current and voltage waveform during the process of charging and discharging periods.

\section{Electromechanical Weak Form Analytical Approach}

The weak form-based Ritz method [50]-[51] reduced from the variational principle is further extended into the proposed system model giving an alternative and direct solution technique. This technique involves a test function in the essence of the piecewise continuous function for the entire structural domain corresponding to virtual relative transverse displacement field, harvesting electrical charge and first and second tuning electrical charges that should meet continuity requirements and boundary conditions. Further detail derivations can be seen in Appendix E. After simplification, the four coupled equations based on the Ritz method-based weak form are formulated as,

$$
\begin{gathered}
\ddot{y}_{r}(t)+2 \zeta_{r} \omega_{r} \dot{y}_{r}(t)+\omega_{r}{ }^{2} y_{r}(t)-\hat{P}_{1 r}^{(1)} P_{1 r}^{(1)} \mu_{1} C_{1} y_{r}(t)-\hat{P}_{2 r}^{(1)} P_{2 r}^{(1)} \mu_{2} C_{2} y_{r}(t) \\
+P_{1 r}^{(1)} \mu_{1} q_{31}^{(1)}(t)+P_{2 r}^{(1)} \mu_{2} q_{32}^{(1)}(t)+P_{r}^{(3)} q^{(3)}(t)=-Q_{r} \ddot{w}_{\text {base }}(t), \\
L_{s 1} \ddot{q}_{31}^{(1)}(t)+R_{1} \dot{q}_{31}^{(1)}(t)+P_{C 1} q_{31}^{(1)}(t)+\hat{P}_{1 r}^{(1)} \mu_{1} y_{r}(t)=0, \\
L_{s 2} \ddot{q}_{32}^{(1)}(t)+R_{2} \dot{q}_{32}^{(1)}(t)+P_{C 2} q_{32}^{(1)}(t)+\hat{P}_{2 r}^{(1)} \mu_{2} y_{r}(t)=0, \\
R_{d} \dot{q}^{(3)}(t)+P_{V}^{(3)} q^{(3)}(t)+\hat{P}_{r}^{(3)} y_{r}(t)=0 .
\end{gathered}
$$


The equations can be further formulated into FRFs as shown in the stage. Moreover, the FRFs provide accurate results as long as the test function-based Ritz eigenfunction is chosen correctly. At this case, since Eq. (38) has been normalized, the parameters $\hat{P}_{1 r}, \hat{P}_{2 r}$ and $\hat{P}_{r}$ can be reduced as,

$$
\hat{P}_{1 r}^{(1)}=\sum_{r=1}^{m} P_{1 r}^{(1)}, \hat{P}_{2 r}^{(1)}=\sum_{r=1}^{m} P_{2 r}^{(1)}, \hat{P}_{r}^{(3)}=\sum_{r=1}^{m} P_{r}^{(3)} .
$$

Note that other parameters can be seen in Appendix E (Eqs. (E11)-(E14)). Laplace transformation can be used to formulate the multi-mode electromechanical FRFs equations giving the transfer functions. Here only one example of the harvesting electrical power FRF is shown across the load resistance after simplification,

$$
\frac{P_{R}^{(3)}(j \omega)}{\left(-\omega^{2} w_{\text {base }} e^{j \omega t}\right)^{2}}=\frac{-\omega^{2} R_{d}\left[\sum_{r=1}^{m} \frac{Q_{r} P_{r}^{(3)}}{N_{r} G}\right]^{2}}{\left[1-\sum_{r=1}^{m} \frac{P_{1 r}^{(1)^{2}} \mu_{1}^{2}}{N_{r} E_{1}}-\sum_{r=1}^{m} \frac{P_{2 r}^{(1)^{2}} \mu_{2}^{2}}{N_{r} E_{2}}-\sum_{r=1}^{m} \frac{P_{r}^{(3)^{2}}}{N_{r} G}\right]^{2}},
$$

where $N_{r}=\omega_{r}{ }^{2}-P_{1 r}^{(1)^{2}} \mu_{1} C_{1}-P_{2 r}^{(1)^{2}} \mu_{2} C_{2}-\omega^{2}+j 2 \zeta_{r} \omega_{r} \omega$ and other parameters of $E_{1}, E_{2}$ and $G$ can be seen in similar forms in (29a)-(29b).

\section{Result and Discussion}

In this section, adaptive tuning piezoelectric harvesting responses were discussed using the two segmented thin electrodes-based shunt circuit control systems. The power FRFs and time waveform DC output responses presented here use the most effective and feasible parametric tuning circuit systems. The material properties of the piezoelectric bimorph beam are given in Table 1. The piezoelectric material used here was made from PZT PSI-5A4E. Note that the input base excitation onto the smart structure was set to be $1 \mathrm{~m} / \mathrm{s}^{2}$. The geometrical structures with tip mass offset as shown in Fig. 1 with beam length $L$ and width $b$ with the lower piezoelectric thickness $h^{(1)}$, substructure (brass) thickness $h^{(2)}$ and upper piezoelectric thickness $h^{(3)}$ were set to $60 \mathrm{~mm}, 6 \mathrm{~mm}, 0.267 \mathrm{~mm}, 0.5 \mathrm{~mm}$ and $0.508 \mathrm{~mm}$, respectively. The dimensions of the tip mass offset $l_{t}, h_{t}$ and $b$ (width) were set to $15 \mathrm{~mm}, 10 \mathrm{~mm}$ and $6 \mathrm{~mm}$, respectively.

Note that due to very low inherent piezoelectric capacitance and lower frequency, large inductance values are required for both tuning circuits. However, as mentioned previously, for practical case, this can be tackled using synthetic inductances which is an integrated circuit mainly consisting of op-amp systems, resistors and capacitor circuits. It is noted that most of the typical power harvesting under load resistance at the first mode shows only one peak of resonance that can shift from short to open circuit resonance. However, after carefully exploring certain tuning circuit parameter values; for our proposed technique, three simultaneous resonances appear to the system followed by widening of the resonant frequency range to more than $10 \mathrm{~Hz}$. Starting with the given example in Fig. 3, using two different electrode segment lengths, the power harvesting FRFs show different trends because contribution of partial electrodes covered onto the piezoelectric layer also indicates certain values of inherent piezoelectric capacitance to the first and second tuning circuits as shown in Fig. 1 giving the options to find the most feasible power harvesting response. As shown, the comparison between electromechanical closed form and Ritz method-based weak form analytical approach gives good agreement. It is noted here that the accurate results given from Ritz method is achieved due to using the same mode shape as given from the closed-form method. Also note that the tuning circuit parameters and the total length of two electrodes of $60 \mathrm{~mm}$ remain constant so as to visualize the effect of tuning electrode segments to the power harvesting circuit. Since power harvesting with electrodes lengths $L_{1}=20 \mathrm{~mm}$ and $L_{2}=40 \mathrm{~mm}$ provides better responses, the widening frequency band and time waveform using variable system parameter will be further explored in the next stage using similar electrode lengths associated with their tuning and harvesting circuit parameter values. In Fig. 4, the three peaks of power harvesting resonances can be visualized using variable harvesting load resistance. Two maximum peaks around $47.3 \mathrm{~Hz}-57.3 \mathrm{~Hz}$ occur not only at the lower load resistances, but also at the higher load resistances. One additional peak showing the lower value can be seen at the lower resonance of $41.7 \mathrm{~Hz}$. Note that the power harvesting FRF with $140 \mathrm{k} \Omega$ in Fig. 4 can also be seen similarly in Fig. 3 (red line) giving the most effective response. Slightly different parametric system behaviour can be seen in Fig. 5a, where shifting resonances from the two maximum peaks 
to single maximum peak appear at the lower and higher tuning circuit load resistances, respectively. A minimum peak at the lower resonance can also be seen. In Fig. 5b, the power harvesting FRF appears to give different trend. It can be remarked in Fig. 5 that the use of the first tuning load resistance connected at the first electrode segment gives a more responsive system compared with the second tuning load located at the second electrode segment.

Table 1. Material properties of the piezoelectric bimorph

\begin{tabular}{lll}
\hline Material properties & Piezoelectric layers & Brass \\
\hline Young's modulus, $\bar{c}_{11}(\mathrm{GPa})$ & 66 & 105 \\
Density, $\rho\left(\mathrm{kg} / \mathrm{m}^{3}\right)$ & 7800 & 9000 \\
Piezoelectric constant, $d_{31}(\mathrm{pm} / \mathrm{V})$ & -190 & - \\
Permittivity, $\varepsilon_{33}^{T}(\mathrm{~F} / \mathrm{m})$ & $1800 \varepsilon_{o}$ & - \\
Permittivity of free space, $\varepsilon_{\mathrm{O}}(\mathrm{pF} / \mathrm{m})$ & 8.854 & - \\
\hline
\end{tabular}

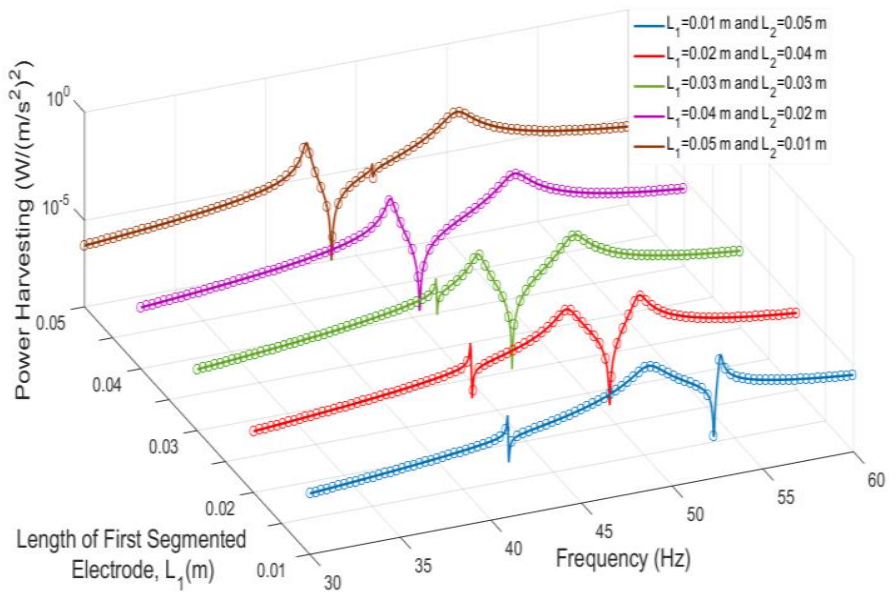

Fig.3. Power harvesting FRFs with different segmented electrode lengths with fixed harvesting load resistance $R_{d}=140 \mathrm{k} \Omega$, fixed first and second tuning capacitors $\mathrm{C}_{1}=25 \mathrm{nF}$ and $\mathrm{C}_{2}=45 \mathrm{nF}$, fixed first and second synthetic inductances $L_{s 1}=330 \mathrm{H}$ and $L_{s 2}=250 \mathrm{H}$, and fixed first and second tuning load resistances $R_{1}=50 \Omega$ and $R_{2}$ $=50 \Omega$ (solid line-closed form and circle-Ritz method).

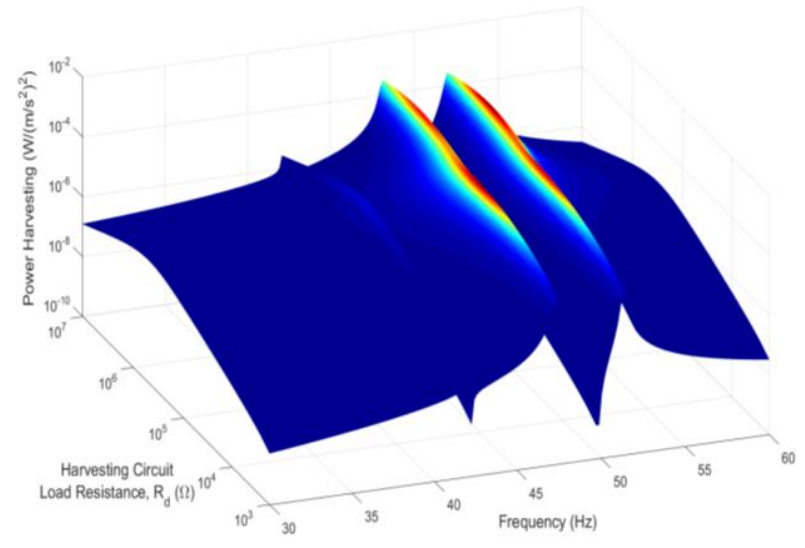

Fig.4. Power harvesting FRFs under varying harvesting load resistance fixed first and second tuning capacitors $C_{1}=25 \mathrm{nF}$ and $C_{2}=45 \mathrm{nF}$, fixed first and second synthetic inductances $L_{s 1}=330 \mathrm{H}$ and $L_{s 2}=250 \mathrm{H}$, and fixed first and second tuning load resistances $R_{1}=50 \Omega$ and $R_{2}=50 \Omega$. 

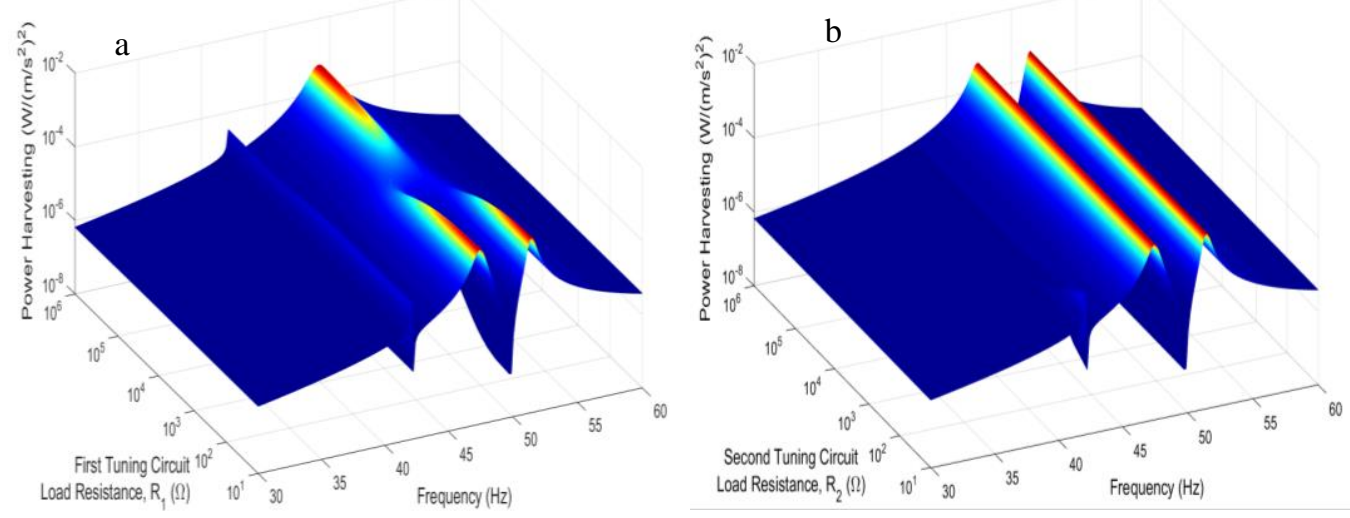

Fig.5. Power harvesting FRFs with fixed first and second tuning capacitors $C_{1}=25 \mathrm{nF}$ and $C_{2}=45 \mathrm{nF}$, fixed first and second synthetic inductances $L_{s 1}=330 \mathrm{H}$ and $L_{\mathrm{s} 2}=250 \mathrm{H}$, and harvesting load resistance $R_{d}=140 \mathrm{k} \Omega:$ a) variable first tuning load resistance with fixed second tuning load resistance $R_{2}=50 \Omega, \mathrm{b}$ ) variable second tuning load resistance with fixed first tuning load resistance $R_{1}=50 \Omega$.
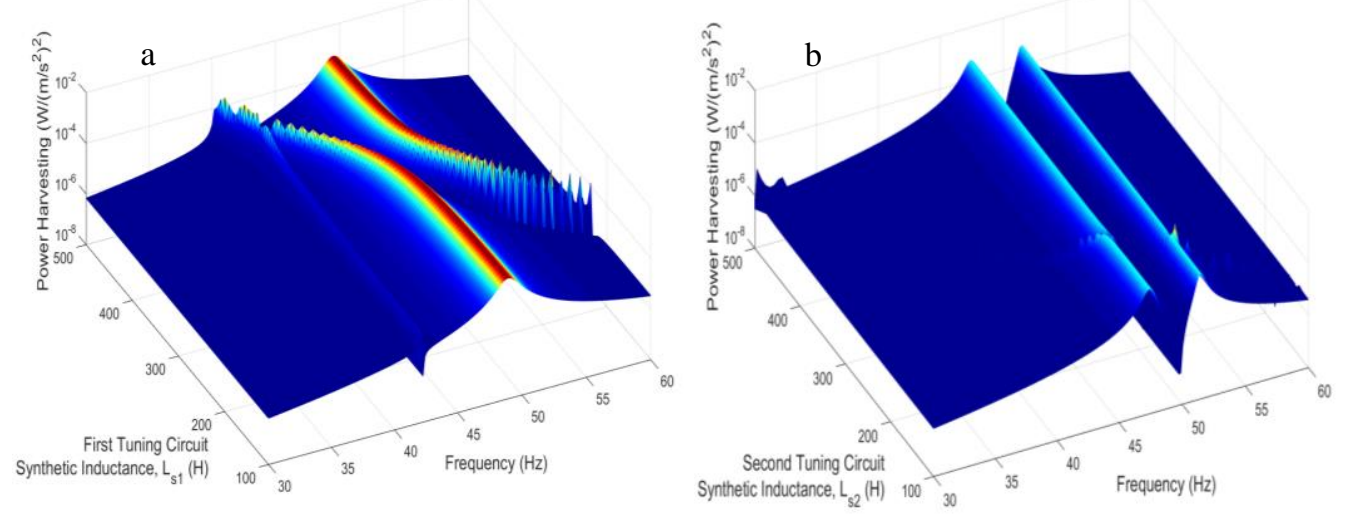

Fig.6. Power harvesting FRFs with fixed first and second tuning capacitors $C_{1}=25 \mathrm{nF}$ and $C_{2}=45 \mathrm{nF}$, fixed first and second tuning load resistances $R_{1}=50 \Omega$ and $R_{2}=50 \Omega$, and harvesting load resistance $R_{\mathrm{d}}=140 \mathrm{k} \Omega$ : a) variable first synthetic inductance with fixed second synthetic inductance $L_{s 2}=250 \mathrm{H}, \mathrm{b}$ ) variable second synthetic inductance with fixed first synthetic inductance $L_{s 1}=330 \mathrm{H}$.
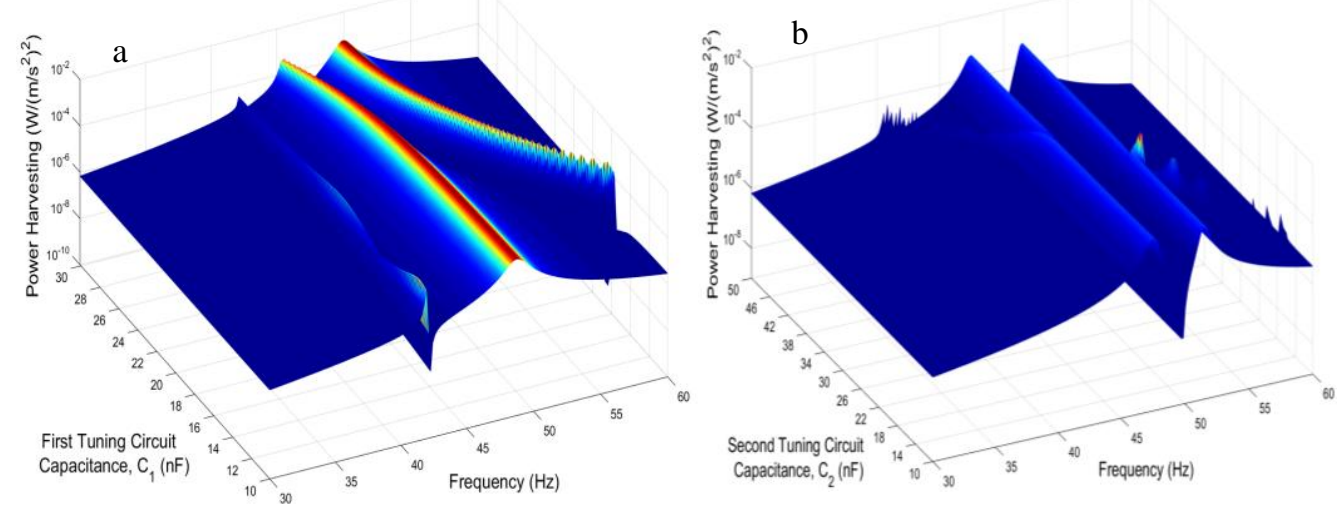

Fig.7. Power harvesting FRFs with fixed first and second synthetic inductances $L_{\mathrm{s} 1}=330 \mathrm{H}$ and $L_{\mathrm{s} 2}=250 \mathrm{H}$, fixed first and second tuning load resistances $R_{1}=50 \Omega$ and $R_{2}=50 \Omega$, and harvesting load resistance $R_{\mathrm{d}}=140 \mathrm{k} \Omega$ : a) variable first tuning capacitor with fixed second tuning capacitor $C_{2}=45 \mathrm{nF}, \mathrm{b}$ ) variable second tuning capacitor with fixed first tuning capacitor $C_{1}=25 \mathrm{nF}$.

This situation occurs because the strain field to induce the polarity of the piezoelectric component predominantly reacts at the certain location quite close to the support of the cantilevered beam (strain node). However, even though the second tuning element is a slightly less responsive system, it can tune the third peak of resonance and also can be used to select the best tuning load resistance values. Moreover, a wider frequency response band as shown in Fig. 6a can also be intensified using the most feasible particular inductance values. At particular inductance values, the three peaks of resonances can be tuned to give even wider frequency range achieving more than $20 \mathrm{~Hz}$. Again, the first tuning inductance value in Fig. 6a provides a more responsive parameter compared with the second tuning element in Fig. 6b. Nevertheless, the required second 
tuning system is an essential feature and complementary to intensify the third peak of the frequency regime as shown in both figures. Like the tuning inductance parameter, another key aspect to optimize the power harvesting FRFs can be seen in Fig. 7a, where the first tuning circuit capacitance can also contribute to widen and intensify the three peaks of the resonances. Moreover, Fig. $7 \mathrm{~b}$ also shows the three peaks, but does not further boost the amplitude response. However, it certainly provides important information for identifying the best value of the second tuning capacitance so as to create the three most feasible peaks of the resonances when analyzing the whole system. Again, the power harvesting FRF with $25 \mathrm{nF}$ and $45 \mathrm{nF}$ for the first and second tuning capacitances in Fig. 7 can also be seen similarly to that in Fig. 3 (red line). As can be seen for the whole scenario so far, the circuit parameters can dependently affect the system of the adaptive power harvesting responses.

The time waveform of the DC electrical output through the rectifier and capacitor at the harvesting circuit can be visualized using tuning circuit parameters and the excited resonance frequency of $51.3 \mathrm{~Hz}$. Note that the resonance of the smart structure appears due to careful selection of the tuning circuit parameters. It should also be noted if the smart structure has similar resonance value with the tuning circuit system resulting in the lowest power amplitude, it will not give benefit for power harvesting application, but rather for vibration suppression. In Fig. 8a, the DC voltage signal amplitudes through the rectifier and capacitor can be seen to have different trend. In the harvesting circuit, the $\mathrm{AC} / \mathrm{DC}$ rectifier with smoothing $R_{d} C_{d}$ circuit has been used for $\mathrm{DC}$ ripple voltage signal behaviours. The process of maintaining the DC voltage level depends not only on the smoothing capacitor $C_{d}$, but also on the value of resistance across the harvesting circuit. This situation will occur using larger harvesting load resistance $R_{d}$ where the time of the capacitor $C_{d}$ to discharge will be short. Note that the charging process through the capacitor only occurs for the ripple process each time the diodes conduct to capture the process of AC-DC current (equation 32). Once the capacitor discharges, no current will flow (equations 33 and 34) as shown in Fig. 8b. It is also obvious to see that the capacitor connected into the full-wave rectifier can only be charged for each half-cycle of the DC signal and then again for the next half-cycle. The prediction of the DC power harvesting time waveform as shown in Fig. 8c can be obtained across load resistance. The fluctuated DC power amplitude provides higher value due to using selective circuit parameters for tuning particular frequency of the system where the performance can also be seen similarly in Fig. 3 (red line). Note that the three peaks of resonances can be the best option for exciting the smart structure so as to maximize the time waveform DC power output. At this case, careful selection of harvesting and tuning circuit parameters can potentially tune and widen the frequency band with increasing power harvester amplitude.
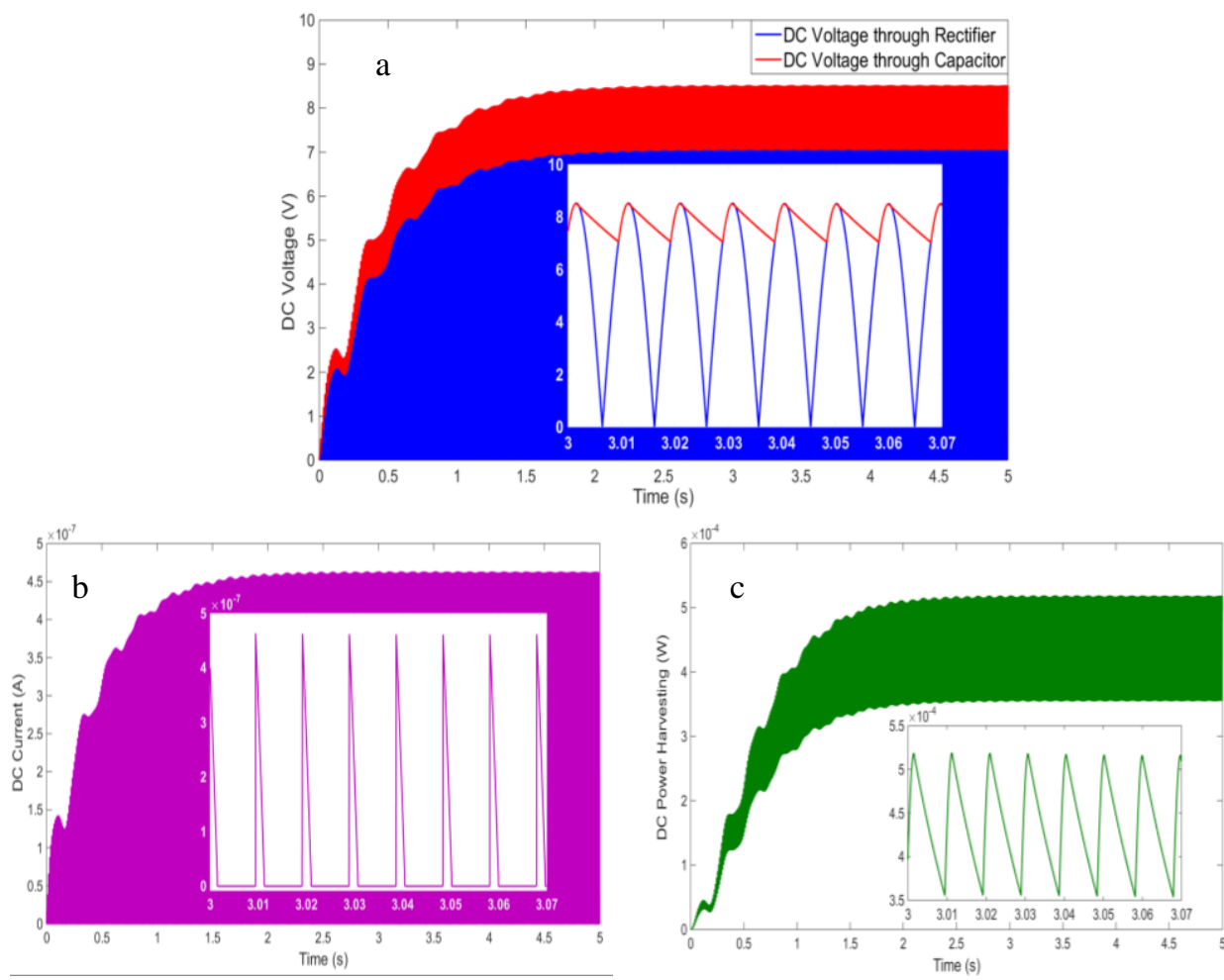

Fig.8. Time waveform signal based on fixed harvesting load resistance $R_{d}=140 \mathrm{k} \Omega$ and capacitance $C_{d}=0.3 \mathrm{nF}$, fixed first and second tuning capacitors $C_{1}=25 \mathrm{nF}$ and $C_{2}=45 \mathrm{nF}$, fixed first and second synthetic inductances $L_{s 1}=330 \mathrm{H}$ and $L_{s 2}=250 \mathrm{H}$, and fixed first and second tuning load resistances $R_{1}=50 \Omega$ and $\left.R_{2}=50 \Omega: \mathrm{a}\right) \mathrm{DC}$ Voltage, b) DC capacitor current, c) DC power harvesting across harvesting load resistance. 


\section{Conclusion}

This paper discussed shunt circuit networks connected to the two segmented electrodes covered onto the bottom surface of the piezoelectric layer for controlling the upper layer of the piezoelectric power harvester. The system provides the adaptive smart structure power harvester to be capable of not only tuning across a certain frequency band but also creating three peaks of the resonances. The electromechanical closed-form boundary value method reduced from the extended Hamiltonian principle was developed to formulate new electromechanical frequency response functions and time waveform systems of the standard AC-DC circuit power harvesting using Laplace transforms. The Ritz method-based weak form analytical approach has also been formulated to give electromechanical transverse dynamic equations for formulating multi-mode FRFs. These two analytical techniques developed coupled systems of the electromechanical power harvesting equations showing the simultaneous combinations of the mechanical system (dynamical behaviour of piezoelectric structure), electromechanical system (electrical piezoelectric response) and electrical system (tuning and harvesting circuits). The two methods have shown identical results in the frequency analysis. The accuracy of the Ritz method was achieved due to using the same mode shape as given from the closed-form boundary value techniques. Moreover, after carefully exploring certain tuning and harvesting circuit parameter values, the results show that varying different electrode lengths connected to the two separated tuning circuits can provide the guideline to identify not only levels of the wider frequency band, but also surveys of the peaks of resonances. For that reason, using particular electrode lengths and tuning and harvesting circuit parameter values, further proofs have been provided using the effect of varying circuit parameters of the system for exploring the widening and increasing the resonance frequency band and time waveform. As a result, adaptive power harvesting techniques can give benefit for tuning frequency response and time waveform in applications of the self-powered wireless sensor devices that is normally located at the surrounding vibration environment for capturing mechanical energy and converting it into the usable electrical energy.

\section{A Appendix: Modified Elastic Constant and Piezoelectric Constant}

The modified elastic constant and modified piezoelectric constant for tuning and harvesting piezoelectric layers can be formulated, respectively as,

$$
\begin{aligned}
& \bar{c}_{D}^{(1)}=\bar{c}_{11}^{(1, E)}+e_{31}^{(1)^{2} \varepsilon_{33}^{(1, S)^{-1}},} g_{31}^{(1)}=\varepsilon_{33}^{(1, S)^{-1}} e_{31}^{(1)}, \\
& \bar{c}_{D}^{(3)}=\bar{c}_{11}^{(3, E)}+e_{31}^{(3)^{2}} \varepsilon_{33}^{(3, S)^{-1}}, g_{31}^{(3)}=\varepsilon_{33}^{(3, S)^{-1}} e_{31}^{(3)} .
\end{aligned}
$$

Note that general parameter $\varepsilon_{33}^{(i, S)}$ for piezoelectric layers (superscript $i \in\{1,3\}$ ) indicates the permittivity at constant strain (superscript $S$ ) that can be further formulated as $\varepsilon_{33}^{(i, S)}=\varepsilon_{33}^{(i, T)}-e_{31}^{(i)} d_{31}^{(i)}$ or $\varepsilon_{33}^{(i, S)}=\varepsilon_{33}^{(i, T)}-d_{31}^{(i)^{2}} c_{11}^{(i, E)}$ where $\varepsilon_{33}^{(i, T)}$ is the permittivity at constant stress (superscript T). Parameter $e_{31}$ is piezoelectric coefficient which is obtained using $e_{31}=d_{31} \bar{c}_{11}^{E}$.

\section{B Appendix: Modified Transverse Piezoelectric Coupling Coefficient}

The modified transverse piezoelectric coupling in the tuning and harvesting piezoelectric layers can be formulated, respectively as,

$$
\begin{gathered}
\eta_{1}^{(1)}=\frac{g_{31}^{(1)}\left(h^{(1)^{2}}+2 h^{(1)} h^{(3)}+2 h^{(2)} h^{(1)}-2 z_{n} h^{(1)^{2}}\right)}{2 L_{1}}, \\
\eta_{2}^{(1)}=\frac{g_{31}^{(1)}\left(h^{(1)^{2}}+2 h^{(1)} h^{(3)}+2 h^{(2)} h^{(1)}-2 z_{n} h^{(1)^{2}}\right)}{2 L_{2}}, \eta^{(3)}=\frac{g_{31}^{(3)}\left(2 z_{n} h^{(3)}-h^{(3)^{2}}\right)}{2 L} .
\end{gathered}
$$

C Appendix: Modified Internal Capacitance of Piezoelectric

The modified internal capacitances in the tuning and harvesting piezoelectric layers can be stated, respectively as,

$$
C_{v 1}^{(1)}=\frac{\varepsilon_{33}^{S} b^{(1)} L_{1}}{h^{(1)}}, \quad C_{v 2}^{(1)}=\frac{\varepsilon_{33}^{S} b^{(1)} L_{2}}{h^{(1)}}, C_{v}^{(3)}=\frac{\varepsilon_{33}^{S} b^{(3)} L}{h^{(3)}} .
$$




\section{Appendix: Mode shapes of the Cantilevered Bimorph Beam with Proof Mass Offset}

The normalized eigenfunction series $\hat{W}_{r}(x)$ in (24) can be proved by manipulating (21) and (23) and taking only consideration of the transverse mechanical equation of using $w(x, t)=\sum_{r=1}^{\infty} w_{r}(x) w_{r}(t)$. The reduced characteristic mechanical equation can be formulated to give,

$$
\left[\begin{array}{ll}
A_{11} & A_{12} \\
A_{21} & A_{22}
\end{array}\right]\left\{\begin{array}{l}
a_{1} \\
a_{4}
\end{array}\right\}=0,
$$

where:

$$
\begin{aligned}
& A_{11}=-(\cos (\alpha L)+\cosh (\alpha L))+\frac{I_{2}^{t i p} \alpha^{3}}{I_{0}}(\sin (\alpha L)+\sinh (\alpha L))-\frac{x_{c} I_{0}^{t i p} \alpha^{2}}{I_{0}}(\cos (\alpha L)-\cosh (\alpha L)) \\
& A_{12}=(\sin (\alpha L)+\sinh (\alpha L))+\frac{I_{2}^{t i p} \alpha^{3}}{I_{0}}(\cos (\alpha L)-\cosh (\alpha L))+\frac{x_{c} I_{0}^{t i p} \alpha^{2}}{I_{0}}(\sin (\alpha L)-\sinh (\alpha L)) \\
& A_{21}=(\sin (\alpha L)-\sinh (\alpha L))+\frac{I_{0}^{t i p} \alpha}{I_{0}}(\cos (\alpha L)-\cosh (\alpha L))-\frac{x_{c} I_{0}^{t i p} \alpha^{2}}{I_{0}}(\sin (\alpha L)+\sinh (\alpha L)) \\
& A_{22}=(\cos (\alpha L)+\cosh (\alpha L))-\frac{I_{0}^{t i p} \alpha}{I_{0}}(\sin (\alpha L)-\sinh (\alpha L))-\frac{x_{c} I_{0}^{t i p} \alpha^{2}}{I_{0}}(\cos (\alpha L)-\cosh (\alpha L)) .
\end{aligned}
$$

The frequency equation and eigenvalues can be formulated from Eq. (D1) leading to nontrivial solutions as,

$$
A_{11} A_{22}-A_{21} A_{12}=0
$$

The mode shape or space-dependent eigenfunction of transverse bending can be formulated can be formulated as,

$$
W_{r}(x)=a_{1 r}\left(\cos (\alpha x)-\cosh (\alpha x)+\frac{A_{21}}{A_{22}}(\sin (\alpha x)-\sinh (\alpha x))\right) .
$$

Since Eq. (D4) contains constant $a_{1 r}$ as the transverse amplitude constant, the normalized mode shape can be formulated as,

$$
\hat{W}_{r}(x)=\frac{W_{r}(x)}{\left(\int_{0}^{L} I_{0} W_{r}(x)^{2} d x+I_{0}^{t i p} W_{r}(L)^{2}+2 x_{c} I_{0}^{t i p} W_{r}(L) \frac{\mathrm{d} W_{r}}{\mathrm{~d} x}(L)+I_{2}^{t i p}\left(\frac{\mathrm{d} W_{r}}{\mathrm{~d} x}(L)\right)^{2}\right)^{1 / 2}}, r=1,2, \ldots, m .
$$

\section{E Appendix: Derivations of Ritz Method-Based Analytical Weak Form}

Further derivations of the electromechanical weak form can be formulated by substituting (7)(13) associated with (16), (17) and (20) into (6) and the result of which can be reformulated by using the normalized eigenfunction series forms, $w(x, t)=\sum_{r=1}^{m} \hat{Y}_{r}(x) y_{r}(t)$. Note that the chosen normalized mode shape-related Ritz method can be discussed in the next stage.

After simplification, the first electromechanical dynamic equation represents the coupled tuning-harvesting piezoelectric bimorph under transverse bending form as,

$$
\begin{aligned}
& \sum_{q=1}^{m}\left\{\sum _ { r = 1 } ^ { m } \left[\left(\int_{0}^{L} I_{0} \hat{Y}_{q}(x) \hat{Y}_{r}(x) d x+I_{0}^{t i p} \hat{Y}_{q}(L) \hat{Y}_{r}(L)+x_{c} I_{0}^{t i p} \frac{\mathrm{d} \hat{Y}_{r}(L)}{\mathrm{d} x} \hat{Y}_{q}(L)+x_{c} I_{0}^{t i p} \hat{Y}_{r}(L) \frac{\mathrm{d} \hat{Y}_{q}(L)}{\mathrm{d} x}\right.\right.\right. \\
& \left.\left.+I_{2}^{t i p} \frac{\mathrm{d} \hat{Y}_{q}(L)}{\mathrm{d} x} \frac{\mathrm{d} \hat{Y}_{r}(L)}{\mathrm{d} x}\right) \ddot{w}_{r}(t)+\int_{0}^{L} C_{t} \frac{\mathrm{d}^{2} \hat{Y}_{q}(x)}{\mathrm{d} x^{2}} \frac{\mathrm{d}^{2} \hat{Y}_{r}(x)}{\mathrm{d} x^{2}} w_{r}(t) \mathrm{d} x\right]+\int_{0}^{L}\left[\eta^{(1)} \frac{\mathrm{d}^{2}\left(H(x)-H\left(x-L_{1}\right)\right) \hat{Y}_{q}}{\mathrm{~d} x^{2}} q_{11}^{(1)}(t)\right. \\
& \left.+\eta^{(1)} \frac{\mathrm{d}^{2}\left(H\left(x-L_{1}\right)-H(x-L)\right) \hat{Y}_{q}}{\mathrm{~d} x^{2}} q_{12}^{(1)}(t)+\eta^{(3)} \frac{\mathrm{d}^{2} \hat{Y}_{q}}{\mathrm{~d} x^{2}} q^{(3)}(t)\right) \mathrm{d} x \\
& \left.+\left(\int_{0}^{L} I_{0} \hat{Y}_{q}(x) \mathrm{d} x+x_{c} I_{0}^{t i p} \frac{\mathrm{d} \hat{Y}_{q}(L)}{\mathrm{d} x}+I_{0}^{t i p} \hat{Y}_{q}(L)\right) \ddot{w}_{\text {base }}(t)\right\} \delta w_{q}(t)=0 .
\end{aligned}
$$

The second, third, fourth, fifth and sixth equations represent the electromechanical harvesting piezoelectric, tuning piezoelectric and tuning circuit forms, respectively to give, 


$$
\begin{gathered}
\left\{\sum_{r=1}^{m} \int_{0}^{L} \eta_{1}^{(1)} \frac{\mathrm{d}^{2}\left(H(x)-H\left(x-L_{1}\right)\right) \hat{Y}_{r}(x, t)}{\mathrm{d} x^{2}} \mathrm{~d} x+\left(\frac{\left(H(x)-H\left(x-L_{1}\right)\right)}{C_{v 1}^{(1)}}+\frac{1}{C_{1}}\right) q_{11}^{(1)}(t)-\frac{q_{31}^{(1)}(t)}{C_{1}}\right\} \delta q_{11}^{(1)}(t)=0, \\
\left\{L_{s 1} \ddot{q}_{31}^{(1)}(t)+R_{1} \dot{q}_{31}^{(1)}(t)+\frac{q_{31}^{(1)}(t)}{C_{1}}-\frac{q_{11}^{(1)}(t)}{C_{1}}\right\} \delta q_{31}^{(1)}(t)=0 \\
\left\{\sum_{r=1}^{m} \int_{0}^{L} \eta_{2}^{(1)} \frac{\mathrm{d}^{2}\left(H\left(x-L_{1}\right)-H(x-L)\right) \hat{Y}_{r}(x, t)}{\mathrm{d} x^{2}} \mathrm{~d} x+\left(\frac{\left(H\left(x-L_{1}\right)-H(x-L)\right)}{C_{v 2}^{(1)}}+\frac{1}{C_{2}}\right) q_{12}^{(1)}(t)-\frac{q_{32}^{(1)}(t)}{C_{2}}\right\} q_{12}^{(1)}(t)=0 \\
\left\{L_{s 2} \ddot{q}_{32}^{(1)}(t)+R_{2} \dot{q}_{32}^{(1)}(t)+\frac{q_{32}^{(1)}(t)}{C_{2}}-\frac{q_{12}^{(1)}(t)}{C_{2}}\right\} \delta q_{32}^{(1)}(t)=0 \\
\left\{\sum_{r=1}^{m} \int_{0}^{L} \eta^{(3)} \frac{\mathrm{d}^{2} \hat{Y}(x, t)}{\mathrm{d} x^{2}} \mathrm{~d} x+\frac{q^{(3)}(t)}{C_{v}^{(3)}}+R_{d} \dot{q}^{(3)}(t)\right\} \delta q^{(3)}(t)=0 .
\end{gathered}
$$

In terms of (E1)-(E6), the normalized electromechanical dynamic equation can be further simplified in the matrix form as,

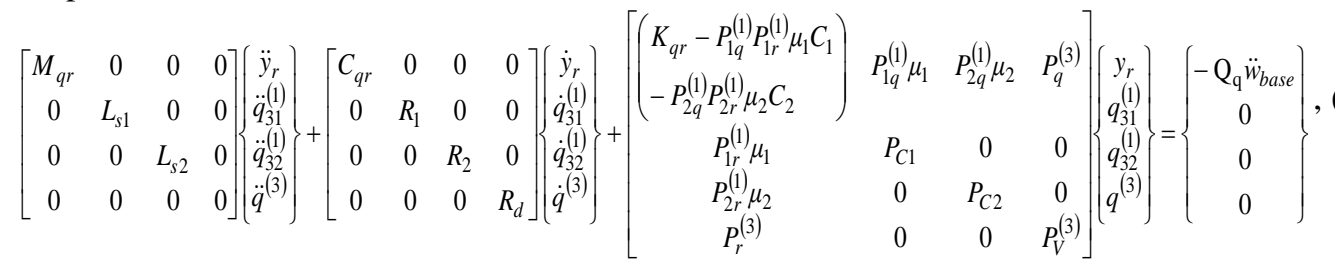

where:

$$
\begin{gathered}
M_{q r}=\int_{0}^{L} I_{0} \hat{Y}_{q}(x) \hat{Y}_{r}(x) \mathrm{d} x+I_{0}^{t i p} \hat{Y}_{q}(L) \hat{Y}_{r}(L)+x_{c} I_{0}^{t i p} \frac{d \hat{Y}_{r}(L)}{d x} \hat{Y}_{q}(L) \\
+x_{c} I_{0}^{t i p} \hat{Y}_{r}(L) \frac{\mathrm{d} \hat{Y}_{q}(L)}{\mathrm{d} x}+I_{2}^{t i p} \frac{\mathrm{d} \hat{Y}_{q}(L)}{\mathrm{d} x} \frac{\mathrm{d} \hat{Y}_{r}(L)}{\mathrm{d} x}, \\
K_{q r}=\int_{0}^{L} C_{t} \frac{\mathrm{d}^{2} \hat{Y}_{q}(x)}{\mathrm{d} x^{2}} \frac{\mathrm{d}^{2} \hat{Y}_{r}(x)}{\mathrm{d} x^{2}} \mathrm{~d} x, P_{1 q}^{(1)}=\int_{0}^{L} \eta_{1}^{(1)} \frac{\mathrm{d}^{2}\left(H(x)-H\left(x-L_{1}\right)\right) \hat{Y}_{q}}{\mathrm{~d} x^{2}} \mathrm{~d} x=\eta_{1}^{(1)} \frac{\mathrm{d} \hat{Y}_{q}\left(L_{1}\right)}{\mathrm{d} x}, \\
P_{2 q}^{(1)}=\int_{0}^{L} \eta_{2}^{(1)} \frac{\mathrm{d}^{2}\left(H\left(x-L_{1}\right)-H(x-L)\right) \hat{Y}_{q}}{\mathrm{~d} x^{2}} \mathrm{~d} x=\eta_{2}^{(1)} \frac{\mathrm{d} \hat{Y}_{q}\left(L-L_{1}\right)}{\mathrm{d} x}, \\
P_{1 r}^{(1)}=\int_{0}^{L} \eta_{1}^{(1)} \frac{\mathrm{d}^{2}\left(H(x)-H\left(x-L_{1}\right)\right) \hat{Y}_{r}(x, t)}{\mathrm{d} x^{2}} \mathrm{~d} x=\eta_{1}^{(1)} \frac{\mathrm{d} \hat{Y}_{r}\left(L_{1}\right)}{\mathrm{d} x}, \\
P_{2 r}^{(1)}=\int_{0}^{L} \eta_{2}^{(1)} \frac{\mathrm{d}^{2}\left(H\left(x-L_{1}\right)-H(x-L)\right) \hat{Y}_{r}}{\mathrm{~d} x^{2}} \mathrm{~d} x=\eta_{2}^{(1)} \frac{\mathrm{d} \hat{Y}_{r}\left(L-L_{1}\right)}{\mathrm{d} x}, \\
\int_{0}^{L} \eta^{(3)} \frac{\mathrm{d}^{2} \hat{Y}_{q}(x)}{\mathrm{d} x^{2}} \mathrm{~d} x, P_{r}^{(3)}=\int_{0}^{L} \eta^{(3)} \frac{\mathrm{d}^{2} \hat{Y}_{r}(x)}{\mathrm{d} x^{2}} \mathrm{~d} x, P_{V}^{(3)}=\frac{1}{C_{v}^{(3)}}, P_{C 1}=\frac{1}{C_{1}}\left(1-\mu_{1}\right), \\
P_{C 2}=\frac{1}{C_{2}}\left(1-\mu_{2}\right), \mu_{1}=\frac{C_{v 1}^{(1)}}{\left(C_{v 1}^{(1)}+C_{1}\right)}, \mu_{2}=\frac{C_{v 2}^{(1)}}{\left(C_{v 2}^{(1)}+C_{2}\right)}, \\
Q_{q}=\int_{0}^{L} I_{0} \hat{Y}_{q}(x) \mathrm{d} x+I_{0}^{t i p} \hat{Y}_{q}(L)+x_{c} I_{0}^{t i p} \frac{\mathrm{d} \hat{Y}_{q}(L)}{\mathrm{d} x} .
\end{gathered}
$$

Here, parameter of the normalized eigenfunction $\hat{Y}_{r}($.$) can be assumed to have a similar form with$ (D5) by considering $\hat{Y}_{r}(x)=\hat{W}_{r}(x)$ and $Y_{r}(x)=W_{r}(x)$. However, parameter $Y_{r}($.$) can be obtained$ from the generalized space-dependent Ritz eigenfunctions as,

$$
Y_{r}(x)=\sum_{k=1}^{m} c_{k r} Y_{k}(x) \quad, \quad r=1,2, \ldots, m
$$


Note that the accuracy of the Ritz mode shape $Y_{k}(x)$ can be obtained using the same mode shape $W_{k}(x)$ as the closed-form boundary value technique where it can be found in (D4) in Appendix D (considering $Y_{k}(x)=W_{k}(x)$ and ignoring constant $a_{1 r}$ because it is used for the closed-form technique where the generalized Ritz method is used in this section). The generalized Ritz coefficient $c_{k r}$ is the eigenvector matrix where each column corresponds to a specific independent eigenvalue. The coefficient can only be proved by replacing $w(x, t)=\sum_{r=1}^{m} \hat{Y}_{r}(x) y_{r}(t)$ with $w(x, t)=\sum_{r=1}^{m} c_{r} Y_{r}(x) e^{i \omega t}$ and rearranging (E7) by considering the characteristic mechanical equation $\sum_{r=1}^{m}\left[K_{q r}-\omega^{2} M_{q r}\right] c_{r}=0, q=1,2, \ldots ., m$. It should be noted that $c_{r}$ is called the Ritz coefficient for the mechanical transverse bending form which sometimes refers to the eigenvectors in the mechanical domain. Corresponding to (E7), the orthonormalizations can now be further proved using the orthogonality property of the mechanical dynamic equations for the Euler-Bernoulli bimorph beam with proof mass offset as,

$$
\begin{gathered}
\int_{0}^{L} I_{0} \hat{Y}_{q}(x) \hat{Y}_{r}(x) \mathrm{d} x+I_{0}^{t i p} \hat{Y}_{q}(L) \hat{Y}_{r}(L)+x_{c} I_{0}^{t i p} \hat{Y}_{q}(L) \frac{\mathrm{d} \hat{Y}_{r}(L)}{\mathrm{d} x}+x_{c} I_{0}^{t i p} \frac{\mathrm{d} \hat{Y}_{q}(L)}{\mathrm{d} x} \hat{Y}_{r}(L)+I_{2}^{t i p} \frac{\mathrm{d} \hat{Y}_{q}(L)}{\mathrm{d} x} \frac{\mathrm{d} \hat{Y}_{r}(L)}{\mathrm{d} x}=\delta_{q r}, \\
\int_{0}^{L} C_{t} \frac{\mathrm{d}^{2} \hat{Y}_{q}(x)}{\mathrm{d} x^{2}} \frac{\mathrm{d}^{2} \hat{Y}_{r}(x)}{\mathrm{d} x^{2}} \mathrm{~d} x=\delta_{q r} \omega_{r}{ }^{2},
\end{gathered}
$$

where $\delta_{q r}$ is the Kronecker delta, defined as unity for $q=r$ and zero for $q \neq r$.

\section{Reference}

1. Roundy, S., Wright, P.K., Rabaey, J.: A study of low level vibrations as a power source for wireless sensor nodes. Comp. Communications, 26(11), 1131-1144 (2003)

2. Chao, P.C-P.: Energy harvesting electronics for vibratory devices in self-powered sensors. IEEE Sensor J. 11, 3106-3121 (2011)

3. Sue, C-Y., Tsai, N-C.: Human powered MEMS-based energy harvest devices, App. Energy 93, 390-403 (2012)

4. Abdelkefi, A., Alothman, A., Hajj, M.R.: Performance analysis and validation of thermoelectric energy harvesters. Smart Mater. Struct. 22, 095014 (2013)

5. Li, Y., Zeynep, C-B., Butler, D.P.: A hybrid electrostatic micro-harvester incorporating inplane overlap and gap closing mechanisms. J. Micromech. Microeng. 25, 035027 (2015)

6. Mann, B.P., Sims, N.D.: Energy harvesting from the nonlinear oscillations of magnetic levitation. J. Sound Vib. 319, 515-530 (2009)

7. Wang, X., Liang, X., Wei, H.: A study of electromagnetic vibration energy harvesters with different interface circuits. Mech. Syst. Signal Process. 58-59, 376 - 398 (2015)

8. Roundy, S., Wright, P.K.: A piezoelectric vibration based generator for wireless electronics. Smart Mater. Struct. 13, 1131-1142 (2004)

9. Erturk, A.: Assumed-modes modeling of piezoelectric energy harvesters: Euler-Bernoulli, Rayleigh, and Timoshenko models with axial deformations. Comp.Struct. 106-107, 214-227 (2012)

10. Lumentut, M.F., Howard, I.M.: Parametric design-based modal damped vibrational piezoelectric energy harvesters with arbitrary proof mass offset: Numerical and analytical validations. Mech. Syst. Signal Process. 68-69, 562-586 (2015)

11. Krommer, M., Irschik, H.: An electromechanically coupled theory for piezoelastic beams taking into account the charge equation of electrostatics. Acta Mech. 154, 141-158 (2002)

12. Krommer, M.: On the correction of the Bernoulli-Euler beam theory for smart piezoelectric beams. Smart Mater. Struct. 10, 668-680 (2001)

13. Irschik, H., Krommer, M., Belyaev, A.K., Schlacher, A.K.: Shaping of piezoelectric sensors/actuators for vibrations of slender beams: coupled theory and inappropriate shape functions. J. Intell. Mater. Syst. Struct. 9, 546-554 (1998)

14. Krommer, M., Zellhofer, M., Heilbrunner, K.-H.: Strain-type sensor networks for structural monitoring of beam-type structures. J. Intell. Mater. Syst. Struct. 20, 1875-1888 (2003)

15. Krommer, M., Irschik, H.: A Reissner-Mindlin-type plate theory including the direct piezoelectric and the pyroelectric effect. Acta Mech. 141, 51-69 (2000) 
16. Krommer, M.: On the influence of pyroelectricity upon thermally induced vibrations of piezothermoelastic plates. Acta Mech.171, 59-73 (2004)

17. Tzou, H.S., Tseng, C.I.: Distributed vibration control and identification of coupled elastic/piezoelectric systems: finite element formulation and applications. Mech. Syst. Signal Process, 5 (3), 215-231 (1991)

18. Krommer, M., Irschik, H.: Sensor and actuator design for displacement control of continuous systems. Smart Struct. Syst. 3, 147-172 (2007)

19. Kapuria, S., Yasin, M.Y.: Active vibration control of smart plates using directional actuation and sensing capability of piezoelectric composites, Acta Mech. 224, 1185-1199 (2013)

20. dell'Isola, F., Maurini, C., Porfiri, M.: Passive damping of beam vibrations through distributed electric networks and piezoelectric transducers: prototype design and experimental validation. Smart Mater. Struct. 13, 299-308 (2004)

21. Niederberger, D., Morari, M.: An autonomous shunt circuit for vibration damping. Smart Mater. Struct. 15 359-364 (2006)

22. Schoeftner, J., Irschik, H.: Passive damping and exact annihilation of vibrations of beams using shaped piezoelectric layersand tuned inductive networks. Smart Mater. Struct. 18, 125008 (2009)

23. Schoeftner, J., Krommer, M.: Single point vibration control for a passive piezoelectric Bernoulli-Euler beam subjected to spatially varying harmonic loads. Acta Mech. 223, 19831998 (2012)

24. Vasques, C.M.A.: Improved passive shunt vibration control of smart piezo-elastic beams using modal piezoelectric transducers with shaped electrodes. Smart Mater. Struct. 21, 125003 (2012)

25. Shu, Y.C., Lien, I.C.: Analysis of power outputs for piezoelectric energy harvesting systems. Smart Mater. Struct. 15,1499-1512 (2006)

26. Guyomar, D., Badel, A., Lefeuvre, E., Richard, C.: Toward energy harvesting using active materials and conversion improvement by nonlinear processing. IEEE Trans. Ultrason. Ferroelectr. Freq. Control. 52(4) 584-595 (2005)

27. Shu, Y.C., Lien, I.C., Wu, W.J.: An improved analysis of the SSHI interface in piezoelectric energy harvesting. Smart Mater. Struct. 16, 2253-2264 (2007)

28. Wang, X., Lin, L.: Dimensionless optimization of piezoelectric vibration energy harvesters with different interface circuits. Smart Mater. Struct. 22(8), 1-20 (2013)

29. Liao, Y., Sodano, H.: Modeling and comparison of bimorph power harvesters with piezoelectric elements connected in parallel and series. J. Intell. Mater. Syst. Struct. 21, 149159 (2010)

30. Kim, M., Hoegen, M., Dugundji, J., Wardle, B.L.: Modeling and experimental verification of proof mass effects on vibration energy harvester performance. Smart Mater. Struct. 19, 045023 (2010)

31. Erturk, A., Inman, D.J.: An experimentally validated bimorph cantilever model for piezoelectric energy harvesting from base excitations. Smart Mater. Struct. 18, 025009 (2009)

32. Lumentut, M.F., Howard, I.M.: Analytical and experimental comparisons of electromechanical vibration response of a piezoelectric bimorph beam for power harvesting. Mech. Syst. Signal Process. 36 (1), 66-86 (2013)

33. Lumentut, M.F. Howard, I.M.: Analytical modeling of self-powered electromechanical piezoelectric bimorph beams with multidirectional excitation, Int. J. Smart Nano Mater. 2 (3), 134-175 (2011)

34. Lumentut, M.F., Howard, I.M.: Electromechanical piezoelectric power harvester frequency response modelling using closed-form boundary value methods. IEEE/ASME Trans. Mechatronics 19, 32-44 (2014)

35. Wickenheiser, A.M.: Eigensolution of piezoelectric energy harvesters with geometric discontinuities: Analytical modelling and validation. J. Intel. Mater. Syst. Struct. 24, 729744 (2013)

36. Lumentut, M.F., Howard, I.M.: Electromechanical finite element modelling for dynamic analysis of a cantilevered piezoelectric energy harvester with tip mass offset under base excitations. Smart Mater. Struct. 23, 095037 (2014)

37. Lumentut, M.F., Howard, I.M.: Intrinsic electromechanical dynamic equations for piezoelectric power harvesters. Acta Mech. DOI: 10.1007/s00707-016-1726-y (2016) 
38. Lumentut, M.F., Francis, L.A., Howard, I.M.: Analytical techniques for broadband multielectromechanical piezoelectric bimorph beams with multifrequency power harvesting. IEEE Trans. Ultrason. Ferroelectr. Freq. Control. 59, 1555-1568 (2012)

39. Lien, I.C., Shu, Y.C. Array of piezoelectric energy harvesting by the equivalent impedance approach. Smart Mater. Struct. 21, 082001 (2012)

40. Xiong, X., Oyadiji, S.O.: Modal optimization of doubly clamped base-excited multilayer broadband vibration energy harvesters. J. Intel. Mater. Syst. Struct. 26(16), 2216-2241 (2015)

41. Wu, P.H., Shu, Y.C.: Finite element modeling of electrically rectified piezoelectric energy harvesters. Smart Mater. Struct. 24, 094008 (2015)

42. Giorgio, I., Culla, A., Del Vescovo, D.: Multimode vibration control using several piezoelectric transducers shunted with a multiterminal network. Arch. App. Mech. 79 859879 (2009)

43. Thomas, O., Ducarne, J., Deu, J.-F.: Performance of piezoelectric shunts for vibration reduction. Smart Mater. Struct. 21, 015008 (2012)

44. Lumentut, M.F., Howard, I.M.: Effect of shunted piezoelectric control for tuning piezoelectric power harvesting system responses - analytical techniques. Smart Mater. Struct. 24 (10), 105029 (2015)

45. Ikeda, T., Fundamentals of piezoelectricity (New York: Oxford University Press, 1990)

46. Tichý. J., Erhart, J., Kittinger, E., Prívratská, J., Fundamentals of piezoelectric sensorics (Springer-Verlag Berlin Heidelberg, 2010)

47. Antoniou, A.: Gyrators using operational amplifiers. Electro. Lett. 3, 350-352 (1967)

48. Riordan, R.H.S.: Simulated inductors using differential amplifiers. Electro. Lett. 3, 50-51 (1967)

49. Moheimani, S.O.R., Fleming, A.J., Piezoelectric transducers for vibration control and damping. (Springer London 2006)

50. Ritz, W.: Über eine neue Methode zur Lösung gewisser Variationsprobleme der mathematischen Physik. Journal für die Reine und Angewandte Mathematik, 135 1-61 (1909)

51. Courant, R., Hilbert, D. Methoden der mathematischen physik / English Ed.: Methods of mathematical physics (New York: Interscience Publishers, 1953-1962) 